\title{
How Can Affect Be Detected and Represented in Technological Support for Physical Rehabilitation?
}

\author{
TEMITAYO A. OLUGBADE, University College London \\ ANEESHA SINGH, University College London \\ NADIA BIANCHI-BERTHOUZE, University College London \\ NICOLAI MARQUARDT, University College London \\ MIN S. H. AUNG, University College London \\ AMANDA C. DE C. WILLIAMS, University College London
}

\begin{abstract}
Although clinical best practice suggests that affect awareness could enable more effective technological support for physical rehabilitation through personalisation to psychological needs, designers need to consider what affective states matter and how they should be tracked and addressed. In this paper, we set the standard by analysing how the major affective factors in chronic pain (pain, fear/anxiety, and low/depressed mood) interfere with everyday physical functioning. Further, based on discussion of the modality that should be used to track these states to enable technology to address them, we investigated the possibility of using movement behaviour to automatically detect the states. Using two body movement datasets on people with chronic pain, we show that movement behaviour enables very good discrimination between two emotional distress levels $(\mathrm{F} 1=0.86)$, and three pain levels $(\mathrm{F} 1=0.9)$. Performance remained high (F1=0.78 for two pain levels) with a reduced set of movement sensors. Finally, in an overall discussion, we suggest how technology-provided encouragement and awareness can be personalised given the capability to automatically monitor the relevant states, towards addressing the barriers that they pose. In addition, we highlight movement behaviour features to be tracked to provide technology with information necessary for such personalisation.
\end{abstract}

CCS Concepts: • Applied computing $\rightarrow$ Life and medical sciences $\rightarrow$ Health informatics; • Human-centred computing $\rightarrow$ Ubiquitous and mobile computing $\rightarrow$ Empirical studies in ubiquitous and mobile computing; - Human-centred computing $\rightarrow$ Human computer interaction (HCI) $\rightarrow$ HCI design and evaluation methods.

\section{KEYWORDS}

Chronic pain, affective computing, affective interaction, physical rehabilitation

\section{ACM Reference format:}

Temitayo A. Olugbade, Aneesha Singh, Nadia Bianchi-Berthouze, Nicolai Marquardt, Min S. H. Aung, and Amanda C. de C. Williams. xxxx. How Can Affect Be Detected and Represented in Technological Support for Physical Rehabilitation? ACM Trans. Comput.-Hum. Interact. xx, xx, Article xx (Month xxx), 28 pages. http://dx.doi.org/10.1145/0000000.

\section{INTRODUCTION}

The growing availability of automatic affect detection capability in the context of healthcare (e.g. in [1-4]) makes it possible to leverage this functionality for personalisation of technological support to affective needs. However, current applications have been limited to substitution of standard questionnaires (e.g. for depressed mood) with the more objective and replicative measure that automatic detection offers. Such a narrow view of the opportunities offered by affect-aware healthcare technology has had a strong influence on how it is investigated and designed: the states tracked, the affective modalities and sensors considered. Design choices are often based on what is most advanced in the affective computing literature, rather than on needs in the application domain. For example, there is a growing body of literature on automatic pain assessment from facial expression [5-9] mirroring consultation settings where the patients sits in front of a clinician or lies on a hospital bed. While this may be useful for clinical consultation, it fails to account for the broader context of and need in the management of long-term medical conditions. In this paper, using the case of self-managed 
chronic pain (CP) physical rehabilitation, we aim to pull together Human-Computer Interaction (HCI) and Affective Computing fields to bring about better understanding of the opportunities for and possibility of affect awareness in healthcare technology.

Rather than implying that affect awareness is necessary for providing effective technological support for physical rehabilitation, this paper aims to explores how synergy between the two fields may inspire reconsideration of design of physical rehabilitation technologies. $\mathrm{CP}$ physical rehabilitation is an interesting case study to investigate this because it mainly takes place outside of clinical settings and in the complexity of everyday life where it is self-managed (i.e. done without a clinician present). This applies to several other conditions requiring long-term physical rehabilitation (e.g. stroke, multiple sclerosis). In addition, although physical rehabilitation beyond clinical settings has received increased attention from technology designers, current designs, such as in [10], rely on models that do not fully represent the multidisciplinary approach used within clinical intervention programs. In best practice, the individual's particular cognitive and affective barriers to movement related to his or her specific goals are addressed by clinicians [11]. This approach comes from the knowledge that reduced engagement in physical activity for people with $\mathrm{CP}$ is strongly predicted by cognitive and affective factors, particularly low movement-related self-efficacy and pain-related fear/anxiety and low/depressed mood, all embedded in the person's beliefs about their pain and experience of trying to manage it [12-13]. The problem, however, is that such tailored interventions are limited to clinical settings and are not feasible during everyday functioning when the challenges arise for the individual; selfmanagement skills and capabilities developed in these programs do not easily transfer to everyday physical activities [14], particularly more challenging ones, often required to achieve valued goals (e.g. employment).

Bringing together three studies based on qualitative methods, machine learning techniques, and wearable prototyping, to better understand the above design space, our main contributions in this paper are as follows:

1. Study 1: Building on the CP literature, we analysed interviews of people with $\mathrm{CP}$ and physiotherapists to generate deeper insights into how pain levels, fear/anxiety, and low/depressed mood interfere with engagement with physical activity in everyday functioning.

2. Study 2: Drawing on findings from Study 1, we investigated the feasibility of automatic detection of emotional distress (fear/anxiety and low/depressed mood as a single construct) and pain levels during physical activity, based on body movement. Body movement was chosen because it is the more helpful modality for understanding pain experience for the purpose of informing support [15]. The pain level detection in Study 2 builds on previous studies (in [16-17]) reported here in more details, for completion.

3. Study 3: To address the reality that physically rehabilitation encompasses both exercise and functional movements, in ubiquitous settings [18-19], we additionally explore the possibility of automatic pain level detection based on a minimal set of low-cost wearable body movement sensors, and in both exercise and functional movements.

4. Finally, we integrate findings from the three studies in a discussion where we highlight opportunities that automatic affect monitoring opens for personalisation of physical rehabilitation technological support, its feasibilities, and HCI and Affective Computing challenges that need to be addressed.

Before reporting these three studies, we first provide a background on $\mathrm{CP}$ and the affective barriers to engagement in physical activity with $\mathrm{CP}$, to clarify the basis for the questions addressed by the studies.

\section{BACKGROUND: CHRONIC PAIN AND ITS AFFECTIVE BARRIERS TO PHYSICAL FUNCTIONING}

Chronic pain (CP) is a prevalent condition [20-21] where pain persists through dysfunctional changes in the nervous system [22], as opposed to ongoing injury. A major consequence of the condition is reduced engagement in valued activities [20][23], influenced by pain as well as fear/anxiety and depressed mood. Pain is a salient signal that interrupts and demands attention [12] and various studies (e.g. [13], [24]) have found 
significant correlation between pain intensity and physical functioning outcomes (avoidance and disability) in people with $\mathrm{CP}$.

But fear/anxiety of movement, pain, or injury contributes to disability independently of pain intensity [24]. The fear-avoidance theory [25] suggests that continued appraisal of pain as a threat causes fear and avoidance of physical activity. In CP, where pain is persistent, avoidance and reduced engagement result in disuse (i.e. loss of muscle fitness) and disability [25]. Nervous system changes further amplify pain, worsening fear of movement and of increased pain [25]. Fear and avoidance may generalise to other activities, exacerbating disability [12], with hypersensitivity to all physical symptoms [25].

The diathesis-stress theory [26] highlights the relevance of depressed mood: living with $\mathrm{CP}$ may, for example, trigger thoughts about the lack of a cure for $\mathrm{CP}$ and such thoughts may provoke a sense of hopelessness and loss of control, which are symptoms of depression. In a sentence completion task, Rusu et al. [27] showed that people with $\mathrm{CP}$ and depressed mood indeed made more health-related completions, and more negatively-valenced completions, than people with $\mathrm{CP}$ without depressed mood. The same study showed that people with $\mathrm{CP}$ with depressed mood reported greater disability and interference of pain with activities than people with $\mathrm{CP}$ without depressed mood.

While this literature clearly identifies pain and related affect as major barriers to $\mathrm{CP}$ physical rehabilitation, the insight is limited as it is based on correlational studies focused on identifying relationships with broad physical functioning outcomes (e.g. disability) assessed using closed-question questionnaire items. Current understanding fails to provide physical rehabilitation technology designers with a strong appreciation of how affective factors interfere with day-to-day physical activities and how affect-based personalisation could enrich technological support. One of the few studies is [11] where initial findings incorporate the perspective of people with $\mathrm{CP}$ in addition to that of physiotherapists. Consistent with the aforementioned studies, the authors suggest that psychological improvement is critical, alongside (or even before) physical progress, in CP physical rehabilitation. Further, the study provides evidence that people with $\mathrm{CP}$ and their physiotherapists adopt strategies to reduce exposure to negative experiences, e.g. careful choice of words in instructions by physiotherapists, with the physiotherapists aiming to help people with CP develop skills to understand and tackle barriers to functional activity. Unfortunately, the findings reported in [11] do not clarify how each of the relevant affective states interferes with variations in physical activity levels, and if (and how) affect-aware capability could inform personalisation of support.

In this paper, we build on the existing knowledge and address the current gap by investigating the questions: 1) what barriers do pain and related fear/anxiety and low/depressed mood present to physical functioning? how should physical rehabilitation technology address the individual states? and 2) can technology detect levels of these states from movement behaviour, to enable personalisation of technological support to these states in everyday physical functioning?

\section{STUDY 1 - HOW AFFECTIVE BARRIERS HINDER EVERYDAY PHYSICAL ACTIVITY IN CHRONIC PAIN}

This study takes advantage of the interview data collected and reported in [11] to provide further understanding of how pain, fear/anxiety, and low/depressed mood influence everyday physical function in people with CP. In [11], due to space, we only reported the variety of barriers to physical activity in $\mathrm{CP}$ and strategies used to facilitate engagement in physical activity, without a deeper discussion of the challenges that these barriers pose and how they can be individually addressed with affect-awareness capability. In this section, we report in-depth analysis of the interviews to understand the distinct barriers that the relevant affective states present and the different forms of support that can address these barriers.

The study was approved by the local research ethics committee and the National Health Service (NHS) research ethics committee, and all participants gave informed consent. The participants were sixteen people with CP (10 women and 6 men, aged 19 to 74 years) and three pain specialist physiotherapists. Each interview lasted between 30 to 45 minutes and was audio recorded. All interviews were transcribed and analysed by the second author (A. S.) using thematic analysis [28]; analysis was iterative until no new concepts emerged. We 
describe the emergent themes in the following subsections. Participant quotes are in italics within double quotation marks, with the respective participant named in the form P\# and PT\# respectively for CP and physiotherapist participants, where '\#' is a numeric identifier.

\subsection{The Effects of Affective Barriers on Engagement in Everyday Physical Activity and The Forms of Personalised Support That Can Address These Barriers}

During our analysis of the data, it became evident that people had strong emotional responses to (the challenge of) and during engagement in physical activity. Three themes were identified: i) pain moderates the value of activity, ii) fear and anxiety can lead to avoiding physical activity, and iii) low mood undermines pain coping and engagement in physical activity. We discuss these themes in this section, highlighting the ways in which support can be tailored to address each of the barriers that they present:

3.1.1 Pain Moderates The Value of Activity. Days where there are pain increases are described as 'bad days', whereas days with lower levels of pain are 'good days'. Some participants with CP reported that they refrained from any physical activity on bad pain days. For P6, "On difficult days, I am paralysed with pain: I'm laid in bed." For others, when pain intensity was high, only highly valued activities could outweigh their desire to rest. Some participants had discovered that lack of activity on bad pain days itself contributed to increased pain, and so had strategies for staying active on those days. P5, for example, said, "I do try to do something because I've found that if I do move about, it helps. I've found that a little bit of movement is better than none". Some chose activities that their experience suggested would lead to pain reduction even if the activities were not of high value. Physiotherapists also stressed the importance of doing a level of physical activity on bad days: "... we try to get them to work out an amount that they can do regardless of if they are having a good or bad day... And that can be anything ... it links back to what their goals are ..." (PT2). Pain levels can also flare up due to the $\mathrm{CP}$ condition, or other triggers such as emotional distress [11] leading to longer setbacks, which take a psychological and physical toll on people with CP. P1 explained, "... if you have a setback for whatever reason, it's easy to assume that you're going to go all the way back to where you started, and this can be very depressing". Physiotherapists agreed that repeated setbacks can be disheartening: "The corrosive effect of repeated flare-ups and that people feel that they're not making progress, that often can mean that people start to slide down into depression, catastrophic thinking, and restrict their activities and interactions." (PT2).

On good days, people tended to overdo physical activity. "On a really good day, I feel like I can do it all, in fact I try to, but I know I shouldn't" (P11). On such days, people could get immersed in activities that they really value and enjoy doing and could end up doing too much. For instance, P3 reported, "When I'm doing it [sewing], I want to finish or get to a point and I forget to [take a] break or [note] how long I've been going and by that time it's too late.". In other cases, people wanted to make up in activities left undone or unfinished on bad days, e.g. catching up on work, or spending time with children. Overdoing activity can lead to normal delayed onset muscle soreness, which may trigger unhelpful rest [29]. Reduced activity following bad days can lead to frustration at reduced capabilities [30-31]. Further, exacerbation of pain following days of overactivity reinforces unhelpful association of pain with physical activity, leading to further reduction of activity [32].

3.1.2 Fear and Anxiety Can Lead to Avoiding Physical Activity. Physiotherapists highlighted fear of pain as the most common deterrent to physical activity for people with CP. PT4 particularly described an experience with a patient: "... a guy I had in clinic who came in absolutely literally scared stiff. This guy cannot move. He cannot forward flex. However, if you sit him down and you reduce levels of fear, he can actually reach down while sitting and touch his ankles. He wouldn't do it when standing because of fear.". This account shows how fear of pain inhibits movements that a person is physically capable of performing. It also demonstrates the importance of context in mediating the relationship between fear and movement. Context may be environmental, e.g. getting up from a low chair versus a high chair, or it may be about the movement itself, e.g. when a movement is one aspect of a complex activity versus when it is part of a less challenging activity. Physiotherapists reported that they often worked with patients to address fear of movement in anxietyprovoking contexts, by increasing the patient's awareness of his/her movement behaviours and abilities while 
performing the movements: “... getting them to recognise it and to really analyse it themselves. People can ... recognise that [they are tense] through relaxation techniques ... as well as cues ... [e.g.] holding their breath ...” (PT3).

Participants with CP also reported that fear of pain exacerbation or injury due to physical activity was reinforced by repeated experience of sudden onset of pain while physically active and led to avoidance of the activity. For example, P12 reported: “... the looking around part of the walking is a challenge and obviously the anticipation of the pain ... I used to go to air shows and look at aeroplanes and obviously that's far more difficult for me now because I can't raise my head ...". Balance or proprioception-related fears [33] were additionally reported to increase caution about certain activities. For P2, "I used to love [it] but I'm so frightened because I've had several bad falls. And I'm frightened of falling over, so I don't do that anymore".

3.1.3 Low Mood Undermines Pain Coping and Engagement in Physical Activity. Some participants highlighted low mood related to living with CP. P2 stated, "... to some degree the pain and the negative outlook in life take over where you just can't be bothered". P12 similarly said, "I devote a fair chunk of my time and thought and energy and day to managing this damn condition ... that is a bit depressing ... it means less time to do things that are actually enjoyable ...". P14 additionally explained, "People will comment, 'But you look so good!' They're like, 'You aren't disabled; why do you have a disabled badge?' It's not obvious, you see, but that little walk in the parking feels to me like running a marathon or the Olympics, every joint hurts." They reported that low activity days were a result not just of increased pain but also of such mood (a term they used interchangeably with 'depression' and 'depressed mood'). On reflection, participants believed that they were more likely to avoid activity when feeling low, irrespective of pain level, for example, finding excuses to avoid chores. P11 particularly said, "Sometimes my mood is quite low and I find it quite difficult to motivate myself. When my mood is better I am able to do more, I feel like doing more." Others reported that it was their ability to cope with pain that was worsened by low or depressed moods. P9 articulated this, "I didn't realize how much my mood affected my pain; the pain is not worse or better but my tolerance is. If I'm sad or worried, my brain can't deal with pain. If I'm upset or in a low mood, pain messages interrupt me rather than me dealing with them."

Physiotherapists agreed that negative beliefs could influence mood and they stressed the importance of awareness of unhelpful beliefs about the relationship between pain and physical activity. PT1 recommended, "Teaching them to address their negative beliefs that are not a true reflection of what's actually going on ... and then getting them to change their negative beliefs into positive and getting them to maybe stick it on their screen somewhere so that they are constantly reminded not to go back into those negative thoughts that can affect their mood."

\subsection{The Need for Self-Awareness of Affective Experiences to Support Self-Management}

We further identified an overarching theme on the need for continuous monitoring of affective experiences in everyday life with CP. Participants with CP felt that better awareness and understanding of their affective states and mood patterns would help them to identify potential triggers of emotional distress. For example, P2 said, "the depression: you're up one minute, down the next, and there seems to be a cycle and, well, I don't log it but it's a good idea, why don't I? (laughs) ...". P9 also reflected, "Work was giving me stress, which was giving me pain and because of the pain I was getting more stressed,.... I couldn't break out of that cycle of frustration, and you know all I could do was feel my pain rather than the frustration and it was really good to see that in a graph.". P2, who had previously been diagnosed with depression, said, "I suffered for many years [with depression] and I've learnt to recognise when you're on the slippery slope downwards and ... crawling back out of it.". This participant applied strategies that reduced the impact of low mood based on his recognition of its onset and offset. However, participants expressed that continuous monitoring can be a challenge: "An individual knows best how they're feeling but you can get all that babble in your head that can easily confuse the messages. If technology enables you to clearly see or think about and reflect on how you are feeling or to selfassess, that would be helpful?' (P2). 
XX:6 - T. A. Olugbade et al.

\subsection{Discussion}

In this study, we sought to deepen the understanding of the barriers that pain, fear/anxiety, and low/depressed mood present to physical functioning. Our findings show that each state raises different needs and that fear/anxiety and low/depressed mood are even very individual. Further, the findings suggest that it is critical for technology to address each need appropriately to facilitate engagement in physical activity. People are not always themselves aware of their affective states, especially at times when they should be addressed. On the other hand, self-monitoring is cognitively demanding. Hence, there is the need for technology that is affect-aware, i.e. capable of detecting pertinent states to provide appropriate and individualised support. Our analysis further highlights design opportunities that build on such capability towards addressing the barriers that pain, fear/anxiety, and low/depressed mood pose to engagement in physical activity. Before we provide a discussion of some of these directions, it is important to first understand the feasibility of the automatic detection of these states. In the rest of this section, we discuss an approach to automatic detection that is grounded in our findings and we describe investigations of this approach in the next sections (Section 4-6). We discuss the findings from these further studies with the proposed affect-awareness-based design principles in Section 8.

Can we simply employ available systems for automatic detection of pain level, mood, and fear? The face and voice have been the favoured affect detection modalities [34-35]. However, the face has been shown to often be neutral during painful movement, with pain related expressions usually appearing only at the end [36], possibly in seeking empathy from the instructor. This is consistent with findings in Sullivan et al. [15] where facial and vocal expressions of pain occurred in pain self-report periods after challenging physical activity rather than during. Our findings from Study 1 suggest that fear/anxiety and increased pain-related behaviour need to be detected the instant they arise, to allow personalisation using context and to variations which can occur within short periods. In addition, these affective channels (the face and voice) can only indicate the presence of a barrier (e.g. fear of pain) but not information about the barrier (e.g. what strategy is used to deal with this barrier). Our findings point to the need to monitor (the quality and quantity of) movement for holistic understanding of the affective barriers faced by a person in engaging in a specific activity at a given instance. Rather than this being a mechanistic approach, it is a means to automatically gain insight into individual challenges and coping strategies. It is especially important to emphasize that, whilst there may be similarities in the way people express pain, fear/anxiety or low/depressed mood, what activity it is experienced in, at what level it is experienced, and how it is dealt with are collectively individual. As shown in the findings in Study 1, physiotherapists read such signals and respond appropriately to help the person better manage the condition and engage more optimally in functioning. Advances in wearable sensing technology, particularly inertia measurement units (IMUs), allow continuous tracking of movement behaviour in a person's natural environment. While increase in stress can also generally be captured in physiological signatures [37] which can be recorded using other types of wearable devices, muscle activity patterns may be the most informative about pain experience with respect to physical activity, given its relation to movement (challenges) [36][38-39]. Muscle activity can be tracked using wearable surface electromyography (sEMG) sensors.

This understanding deriving from the findings in Study 1 leads to the second question we addressed in this paper: can pain-related emotional distress (i.e. fear/anxiety and low/depressed mood as a single construct) and levels of pain be automatically detected from movement behaviour and muscle activity? In investigating this question, we distinguish pain from emotional distress and leave differentiation of fear/anxiety from low/depressed mood for future work. The methods we used, and our findings are reported in Sections 5-7 (Studies 2 and 3); but first, we further discuss the state of the art in automatic detection of pain level and emotional distress.

\section{PREVIOUS WORK: AUTOMATIC PAIN AND EMOTIONAL DISTRESS LEVEL DETECTION}

There have been many studies examining automatic detection of levels of pain, although mostly using facial expressions (e.g. recent studies: [5-9]). As earlier discussed, the face provides only a partial understanding of a 
person's state and consequent behaviour or action readiness [15] and is further impractical for affect tracking in mobile contexts. A considerable proportion of studies on automatic pain level detection using body movement have been based on experimentally induced pain (e.g. in [7][40-41]). Studies where pain is experimentally induced have limited application for CP [42] because healthy participants find the pain stimulus only minimally threatening (or even not at all threatening) and momentary [43], whereas clinical pain is usually threatening and, for $\mathrm{CP}$, enduring. More relevant, therefore, are the studies on people with $\mathrm{CP}$. The earliest of these is the work of [44] where features of the activities of the lumbar paraspinal (lower back) muscles and the range of trunk movement during trunk exercises were used to automatically differentiate participants with low back CP from healthy participants. Similarly, [45] used features such as the range and velocity of the neck in neck rotation and flexion exercises to automatically discriminate between participants with neck CP and healthy participants. Another study by [46] considered the amplitude and time of ground force reaction peaks in foot movements for participants with knee $\mathrm{CP}$ while they walked. Although these studies do not further classify participants with $\mathrm{CP}$ according to their levels of pain, their findings provide understanding that how the painful anatomical segment is handled in movement can betray that a person has CP. Indeed, in [47], spinal motion features were used to automatically differentiate 11 levels of pain in people with low back $\mathrm{CP}$ during trunk exercises. A major limitation of the study, however, is that these features were intricate vertebral movements which were captured using sensors mounted on pedicle screws that had been inserted into the spine under general anaesthesia as part of clinical assessment. This capture method is invasive and is not feasible outside of clinical settings. Thus, the study provides limited understanding of how levels of pain may be tracked based on movement behaviour data captured in everyday settings.

Previous work on the automatic detection of mood levels has been limited to the detection of (the severity of) major depressive disorder based on observation in sedentary clinical consultation settings (e.g. [1-3][4853]). For example, in [1], video-based full-body movement features were used to discriminate between two levels of clinical depression. The authors found that the best performance was obtained by combining holistic movement features and features of the relative movement of anatomical segments. In [3], video-based features of upper body movement, head movement, and facial expressions were used to automatically discriminate between healthy participants and participants with clinical depression. It was found that using head movement features alone led to the same performance as the use of facial features alone while the use of upper body movement features alone led to better performance. In [2], the authors used video-based upper body movement and facial features and acoustic features to discriminate between these two groups. They found that the audio features alone generally led to better performance than the video-based features, but the best performance was obtained with fusion of the two modalities. In similar work by [49], video-based head movements alone were used. Further, [51] and [54] used a combination of video-based eye and head movement and facial features, speech acoustics (and transcript in [51]), and features of previous emotion label annotations. Whereas [54] considered automatic detection of 25 levels, [51] considered 2 levels. In CP, painrelated low/depressed mood is more relevant than major depressive disorder [27][55], and so we make that our focus in this paper. Nevertheless, these previous works provide some understanding of how depressed mood may be detected from movement behaviour although their findings are with respect to video-based features that do not easily transfer to other movement sensors such as IMUs.

While automatic fear/anxiety detection has been investigated in more mobile settings, most studies on it have only considered acted expressions, such as in the studies of [35][56-59]. Unfortunately, the findings from these studies are limited as acted expressions do not fully reflect spontaneous expressions in real life because they are usually exaggerated and so more salient. Although Rani et al. [60] investigated the differentiation of spontaneous anxiety from engagement, anger, frustration, and boredom (based on bodily muscle activity, facial, and physiological cues), this was done in sedentary settings and, as with the other studies, their study of fear/anxiety was not in the context of pain. As discussed in Sections 2 and 3, movement is the object of the fear/anxiety in the context of pain and so, fear/anxiety in that context may be differently embedded in the execution of the movement. Aung et al. [36] investigated the automatic detection of specific bodily expressions associated with pain: guarding (stiffness in movement [61]), bracing, rubbing, abrupt motion, and 
limping. However, pilot analysis showed that these observer-rated expressions cannot be simply used as a proxy for self-reported experiences.

Overall, the state of the art in automatic detection does not fully address the affect monitoring needs of CP physical rehabilitation. Thus, in Study 2, we investigated automatic detection of pain-related emotional distress (combined low/depressed mood and fear/anxiety) and pain levels from movement behaviour captured during exercise movements using IMU and sEMG sensors. This study is based on the existing EmoPain dataset [36]. We further investigate in Study 3 the possibility of detecting pain levels during both functional and exercise movements tracked using a limited network of sensors. The latter investigation is the first step in understanding the feasibility of pain level monitoring in everyday functioning (beyond physical exercises), which, as discussed in earlier sections and in [19][62], is a crucial part of CP physical rehabilitation. This investigation is based on a new dataset (the Ubi-EmoPain dataset [63]) purposely collected for this study. For completeness, we include results from our initial studies [16-17] based on exercise movements in the EmoPain dataset with more details of our methods and analyses. In our investigations, effort is put into analysis of movement behaviours so as to inform features for automatic detection. This approach is necessary because, similar to humans [64], the 'mind reading' capabilities of artificial systems rely on inference from perceptible behaviour or biosignals.

\section{STUDY 2 and 3 - THE AUTOMATIC DETECTION OF EMOTIONAL DISTRESS AND PAIN LEVELS - METHODS}

As discussed above, findings in Study 1 lead to the question of the feasibility of automatic detection of pain and emotional distress (fear/anxiety and low/depressed mood) from movement behaviour, with the aim of enabling technology to personalise support so as to address affective barriers that people with $\mathrm{CP}$ face in functioning. Study 2 investigates this question based on a dataset of exercise movements captured using commercial sensors, and Study 3 builds on this with further investigation based on a second dataset containing both exercise and functional movements captured using custom-built low-cost sensors [63].

In this section, we describe the datasets, features, and analysis methods used in Studies 2 and 3 for investigating automatic emotional distress and pain level detection. Rather than aiming to build a complete system for continuously monitoring these affective states, we investigated the possibility of assessing these states in preselected activities that are generally considered challenging by people with $\mathrm{CP}$.

\subsection{Datasets}

Two datasets were used: the first is an existing dataset while the second was built based on the results obtained from investigation with the first dataset. For the sake of organisation of the paper, we describe both datasets here before the results of the investigation based on the first are presented:

5.1.1 EmoPain Dataset. The EmoPain dataset [36] consists of motion capture and muscle activity data collected from healthy control participants and participants with low back CP. The data was acquired while the participants performed physical exercises that simulate everyday physical activities which are challenging for people with low back CP. In our investigations, we focus on three of these exercises: Sit-toStand, Forward Trunk Flexion, and Full Trunk Flexion [39][65]. The motion capture data obtained includes three-dimensional positions of 26 anatomical joints from the crown to the heel using a commercially available system. The muscle activity data was obtained bilaterally from the trapezius and the lumbar paraspinal muscle also using a commercial system.

Healthy participants and participants with CP completed the Hospital Anxiety and Depression Scale (HADS) [66] before performing the exercises. The HADS is a 14-item scale which measures emotional distress [67-68]. In building automatic emotional distress detection functionality in this paper, we used the HADS for ground truth; however, we excluded one item of the scale because of possible somatic interpretations in $\mathrm{CP}$ [69-70]. There were 112, 45, and 16 instances of Sit-to-Stand, Forward Trunk Flexion, and Full Trunk Flexion respectively with corresponding HADS self-reports. Thus, we were not able to explore automatic 
differentiation of all possible scores of the HADS-13 for each exercise type; the lack of sensitivity of these types of questionnaires (despite their standardisation) was an additional challenge. Instead, we defined two levels of emotional distress, not distressed and distressed, based on a near equal split of the scale.

After performing each exercise at a given level of challenge, participants with $\mathrm{CP}$ reported the intensity of pain experienced on a scale of 0 for no pain to 10 for extreme pain [71]. The healthy control participants did not report pain intensity and were assumed to have no pain. There were 104, 49, and 18 instances altogether of the Sit-to-Stand, Forward Trunk Flexion, and Full Trunk Flexion respectively with associated pain intensities. Although there were participants with $\mathrm{CP}$ who reported experiencing no pain during exercise instances, we did not consider them to be in the same pain class as the healthy control participants. Thus, instances of the healthy control group were labelled as no $C P$ separate from the pain levels considered within the group of participants with $\mathrm{CP}$. In the $\mathrm{CP}$ group, two pain levels were considered: we were not able to consider all intensities of pain on the pain scale due to data size constraints. The two levels of pain, lower level and higher level, in the CP group were derived by a near equal split of the pain intensity scale.

5.1.2 Ubi-EmoPain Dataset. Unlike the commercial sensors used to acquire body movement data in the EmoPain dataset, here, custom-built low-cost sensors were used to obtain the Ubi-EmoPain dataset [63]. The purpose of using custom-built sensors was to explore the feasibility of capturing movement behaviour for automatic monitoring using cheaper and lower fidelity sensors. Thus, in building this dataset, we also used a reduced number of sensors: 4 inertial motion capture units placed on the head, trunk, upper leg, and lower leg and 2 sEMG units attached to the right trapezius and lumbar paraspinal muscles respectively. This is a first step in investigating the possibility of mass deployment of automatic pain level monitoring functionality in physical rehabilitation technology. These placements were based on findings from the studies based on the EmoPain dataset reported in Sections 7.1. The studies showed that movement features of these anatomical segments and muscles were necessary for discrimination between levels of pain in people with $\mathrm{CP}$.

As with the EmoPain dataset, the sensing units were used to collect body movement data from people with $\mathrm{CP}$ in the lower back with data recorded during Forward Trunk Flexion, Full Trunk Flexion, and Sit-to-Stand. However, here, there were instances of Forward and Full Trunk Flexion performed as functional movements in addition to those completed as exercises. For functional Forward Trunk Flexion, the participants were instructed to retrieve a cardboard box attached to the wall with pressure-sensitive adhesive; a barrier was placed on the floor in front of the wall to force participants to reach forward to complete the task. For functional Full Trunk Flexion, participants were asked to pick up a cardboard box from the floor starting from standing. For the Sit-to-Stand, the participants were simply asked to stand up as they normally would. Similar to the EmoPain corpus, emotional distress and pain intensity were assessed via self-report, and the pain selfreport was used to derive two pain classes for automatic detection. Only one participant belonged to the distressed class and so further investigation of emotional distress detection based on this dataset was not done. For Forward Trunk Flexion, there were 27 and 21 instances of lower and higher level pain respectively; 27 and 19 instances respectively of lower and higher level pain for Full Trunk Flexion; and 25 and 13 lower and higher level pain instances respectively for Sit-to-Stand.

\subsection{Body Movement Features}

In the following subsections, we describe the features extracted for emotional distress and pain detection. The selection of features was based on relevant literature and physiotherapist-informed analysis of videos from the EmoPain dataset. The formulae used for extracting these features are provided in Appendices A and B respectively.

5.2.1 Emotional Distress Features. Head slump is one of the known cues of emotional distress [72-74] so we extracted the lowest angle of the head and the duration of that head pose (with a $10^{\circ}$ allowance to account for slight movements), normalised to the duration of the activity. A similar cue of emotional distress is shoulder protraction [75] and we also extracted this. [75] computed shoulder protraction in a single pose (rather than over a movement sequence) and their computation method relied on the use of photographs. As the type of data in our investigation differs from theirs, we instead computed shoulder protraction as the 
minimum angle between the left and right sides of the upper trunk. Another cue thought to be indicative of emotional distress is increased use of self-adaptors, i.e. self-touching [73]. [73] considered three forms of selfadaptors: head, hand, and trunk self-adaptors, which are practical in the clinical interview settings they considered. In our case where participants were engaged in physical activity, we did not expect these adaptors and we instead considered thigh self-adaptors. To characterise thigh self-adaptors, we extracted the minimum distance between the hands and the thighs. Body sway has also emerged as a cue through which emotional distress is expressed [76-77]. To quantify body sway, we computed the energies of the head, trunk, hands, and legs. Energy was computed as the sum of the mean translational and rotational kinetic energies of the specific anatomical segment, assuming unit mass for each. Speeds of the trunk, hands, and legs were additionally extracted based on the findings of reduced speed in the emotionally distressed [76][78].

5.2.2 Pain Level Features. Submaximal flexion of the trunk during Forward and Full Trunk Flexion and Sit-to-Stand is one of the known expressions of low back pain [39][44][79-80]. Thus, for each of the three activity types, we extracted the range of trunk flexion as a feature. For the Sit-to-Stand, we additionally extracted knee and pelvic angles at the point of lift to characterise the relation between the height of the subject and the height of the seat as the amount of trunk flexion in this activity type depends on this relation (although these features may also be influenced by the pain-related affective state of a person). Visual inspection of the EmoPain Forward Trunk Flexion data also showed that there was less flexion of the head with respect to the trunk in participants with CP compared with healthy participants and less arm unsteadiness in instances of higher level pain compared with instances of lower level pain. These two findings suggest stiffness in movement due to pain [61] although the two expressions could also be a result of relatively small trunk movements. Based on the findings, we extracted the amount of neck flexion as a feature for the three activity types. In addition, we characterised unsteadiness in arm movements (only for the study based on the EmoPain dataset) as the number, time range of occurrence, and mean amplitude of peaks in the smoothed profile of the vertical displacement of the upper arm. These were extracted for only the Forward and Full Trunk Flexion, which both involve arm movements. For the Sit-to-Stand, we extracted speed of lift and duration (the latter only for the study based on the EmoPain dataset) as a feature, since reduced speed is an expression that has been shown to be indicative of low back pain in this movement [79].
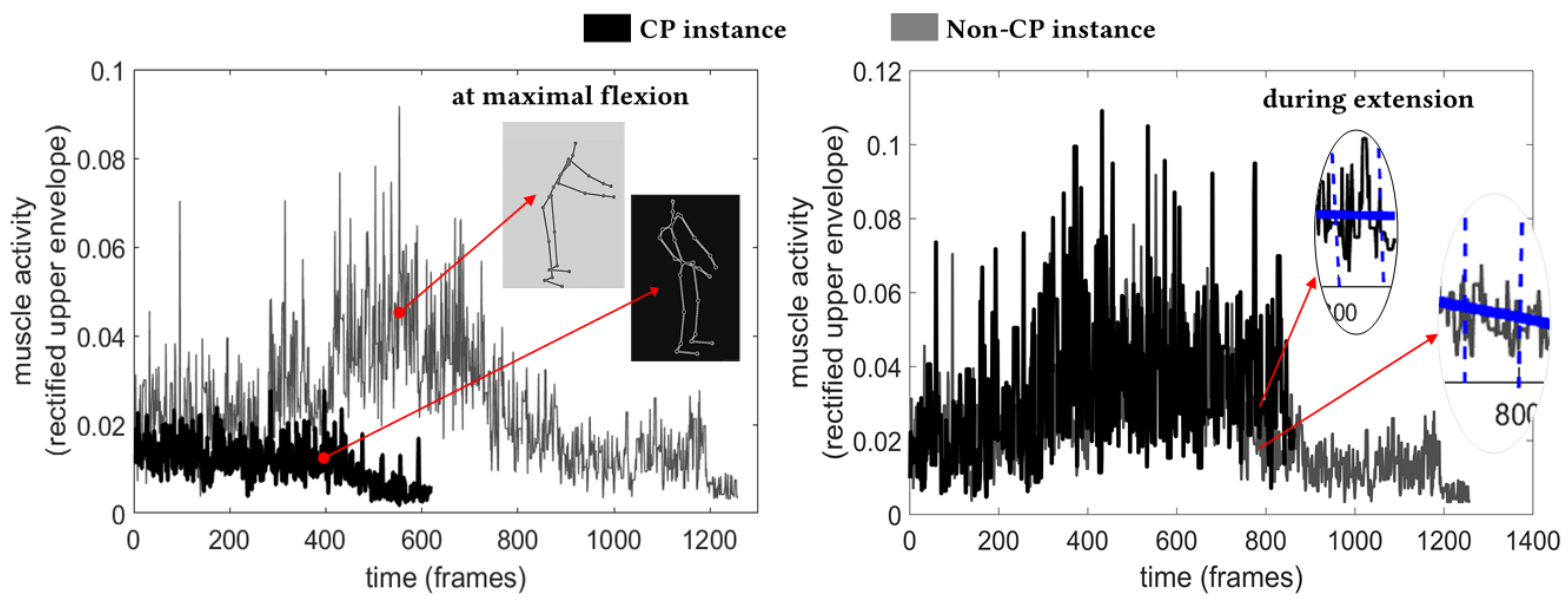

Fig. 1. Left - Minimal activity in the right lumbar paraspinal muscle during Forward Trunk Flexion concomitant with submaximal flexion compared with the healthy pattern; reconstructed skeletons at the top right show the amount of trunk flexion at maximal flexion. Right -

Delayed relaxation in the right lumbar paraspinal muscle on completing re-extension in Forward Trunk Flexion compared with the healthy pattern in the same movement. Both plots are based on the EmoPain dataset. 
Findings from pain studies also show that people with low back $\mathrm{CP}$ have higher lumbar paraspinal muscle activity than healthy participants when fully flexed (typically between $40^{\circ}$ and $70^{\circ}$ of trunk flexion) [39][44]. During this period, the healthy pattern is muscle relaxation in the lumbar paraspinal as the rest of the flexion is achieved by the pelvic muscles [39][44]. Visual inspection (see Fig. 1) based on the EmoPain Forward Trunk Flexion data indicates that there may also be absence (or rather, delay) of re-extension relaxation in the participants with $\mathrm{CP}$ who perform trunk flexion. Thus, we computed both flexion and re-extension relaxation; this was done by modelling muscle activity change point, i.e. the point where higher muscle activity changes to lower activity, based on [81]. Fig. 2 shows the computed location of the change point for the three distinct muscle activity patterns of Fig. 1 which are representative of the EmoPain Forward Trunk Flexion data. For Forward and Full Trunk Flexion, we extracted two features of the change point: the time when it occurs and the amount of change in muscle activity that occurs. Only the former change point feature was extracted for the Sit-to-Stand; for this activity type, the range of muscle activity was also extracted.
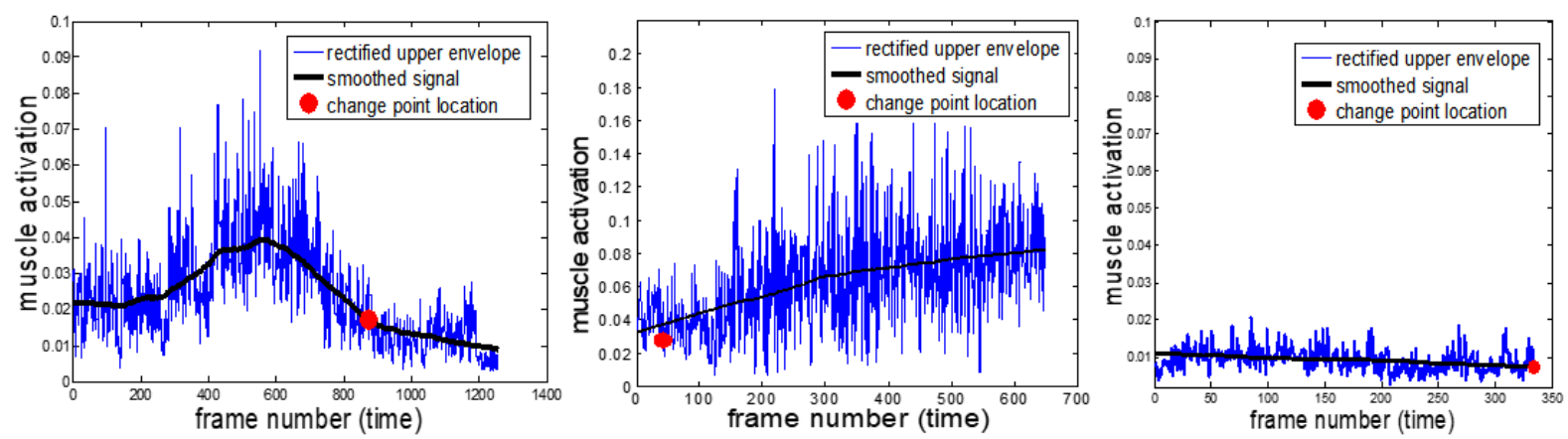

Fig. 2. The location of the computed change point for three different patterns of muscle activity, based on the EmoPain dataset.

\subsection{Classification}

We used standard machine learning classification algorithms in our investigation based on the EmoPain dataset: Support Vector Machine (SVM) and Random Forest (RF). The SVM is a binary classification algorithm that classifies by finding the hyperplane that separates observations into two classes with a maximum margin [82]. In the detection of three pain levels using the SVM (in Study 2), we employed a hierarchical structure of SVMs where an SVM (SVM1) was used to discriminate between CP and non-CP instances and a second SVM (SVM2) was used to further class the CP instances as either lower or higher level pain. The RF, on the other hand, is an ensemble of decision trees that allows for multiclass classification [83]. As is typical in machine learning, we set hyperparameters for both algorithms using grid search.

For the Ubi-EmoPain dataset, $58.4 \%$ of the instances had incomplete feature vectors due to technical malfunction during data capture and inadequate size of an attachment accessory which led to non-capture, in these instances, of any data for some of the anatomical segments. Thus, we explored two approaches for automatic classification with missing data. In one approach, we used single imputation based on linear regression to recover the missing feature values. In the regression model for imputation, we included relevant and available data (the order of the activity instances, pain and HADS-13 scores, and movement confidence self-report also obtained during data collection [63]) as predictor variables. We then used the imputed datasets in building the classification models. In the second approach, we used decision trees with surrogate splits [84] for building the classification model with the incomplete (non-imputed) datasets. To allow for comparison, we also used decision trees for classification with the imputed datasets. Hyperparameter setting was also done here based on grid search. 
$\mathrm{XX:12}$ - T. A. Olugbade et al.

For both the EmoPain and Ubi-EmoPain datasets, we evaluated classification performance using leave-onesubject-out cross-validation, which is standard in affective computing. This method evaluates the generalisability of a classifier to unseen subjects and accounts for idiosyncrasies. We report our results using standard metrics: accuracy as an increasing score between 0 and 1, F1 score (scored similar to accuracy), which quantifies precision and recall with less bias, and confusion matrices, which allow detailed review of classification performance.

\subsection{Features Analysis}

To add to existing knowledge on the movement behaviour cues of emotional distress and pain, we analysed the features extracted from the EmoPain dataset using both feature subset selection and traditional statistics techniques. These methods enable exploration of minimisation of the number of anatomical segments to be tracked for automatic detection, and so the number of sensor units needed for data capture. This would facilitate movement behaviour tracking (for automatic detection) in ubiquitous settings.

We used linear mixed model approach to test for fixed effects of levels of pain and emotional distress on each feature, independent of the classification algorithms used. This method was used as the dataset did not satisfy independence of observations. It was necessary to bootstrap (with the number of samples set to 1000 for bootstrapping) the data for this analysis as the dataset additionally did not satisfy the assumptions of normal distribution and heteroscedasticity of variance [85]. A limitation of the bootstrapped linear mixed model analysis in IBM SPSS (which we used for our analysis) is that only significance of effect is provided without the test statistic.

We used feature subset selection to understand the relevance of the features with respect to the classification algorithm used. This method additionally enabled consideration of more complex interactions between features than the linear mixed model. Our approach to feature subset selection was a breadth-first tree search with pruning similar to the method of [86]. Feature understanding that resulted from feature subset selection was used to optimise classification performance.

\section{STUDY 2 - AUTOMATIC DETECTION OF EMOTIONAL DISTRESS - RESULTS}

In this section, we report the performance of automatic emotional distress detection and the relevance of the selected features (see Section 5.2) to this task.

\subsection{Results: Classification Performance}

We found that using the optimised feature set, the best classification for each of the three activity types was better than chance level performance (see Table 1). For the Forward Trunk Flexion and Sit-to-Stand, the RF outperformed the SVM (although only slightly); however, for the Full Trunk Flexion, the RF did much worse than the SVM and did not recognise distressed level better than chance level detection. As can be seen in Table 2, for the best classifiers, recall of the distressed class was highest in Sit-to-Stand compared with the other two activity types. This may be due to the higher number of training instances for that class available in this activity type in contrast to the other two. Precision for the distressed class was nevertheless perfect for Full Trunk Flexion although the low number of instances in that class makes it difficult to draw any conclusions. In all cases, detection of the distressed level was more difficult than the not distressed level. This is not surprising given the skew in the number of examples of the levels in the dataset, despite using weighted costs and sampling.

\subsection{Results: Features Analysis}

6.2.1 Feature Subset Selection. We analysed the importance of the features extracted based on the frequency of the features in the subsets returned with feature subset selection using the best classifier for each activity type. For the Full Trunk Flexion, the right lower leg energy feature, the minimum head angle, and the 
minimum hand to thigh distance were found to be the most important features. Further analysis (Fig. 3-left) showed that the right lower leg energy was typically less in the distressed group. We found that the energy features were generally useful for the Full Trunk Flexion. For the minimum head angle feature, half of the distressed instances had lower values than nearly three-thirds of the not distressed instances (Fig. 3-middle). The importance of this feature in the Full Trunk Flexion but not the other two activity types may be because, in contrast to these activity types, the Full Trunk Flexion lends itself to head slump. We also found that the distressed group had lower hand to thigh minimum distances than the not distressed (Fig. 3-right).

Table 1. Emotional Distress Level Classification Results (based on Feature Subset Selection)

\begin{tabular}{clccccc}
\hline & \multicolumn{2}{l}{ Full Trunk Flexion } & \multicolumn{2}{c}{ Forward Trunk Flexion } & \multicolumn{2}{c}{ Sit-to-Stand } \\
& SVM & RF & SVM & RF & SVM & RF \\
\hline F1 not distressed & $\mathbf{0 . 9 6}$ & 0.83 & 0.92 & $\mathbf{0 . 9 5}$ & 0.92 & $\mathbf{0 . 9 5}$ \\
F1 distressed & $\mathbf{0 . 8 0}$ & 0.50 & 0.67 & $\mathbf{0 . 7 1}$ & 0.75 & $\mathbf{0 . 7 6}$ \\
average F1 & $\mathbf{0 . 8 8}$ & 0.67 & 0.80 & $\mathbf{0 . 8 3}$ & 0.84 & $\mathbf{0 . 8 6}$ \\
accuracy & $\mathbf{0 . 9 4}$ & 0.75 & 0.87 & $\mathbf{0 . 9 1}$ & 0.88 & $\mathbf{0 . 9 1}$ \\
\hline
\end{tabular}

Table 2. Confusion Matrices for The Best Classifier in Table 1 for Each Activity Type

\begin{tabular}{|c|c|c|c|c|c|c|c|}
\hline & \multicolumn{2}{|c|}{$\begin{array}{c}\text { FULL FLEXION } \\
\text { Automatic Detection }\end{array}$} & \multicolumn{2}{|c|}{$\begin{array}{l}\text { FORWARD FLEXION } \\
\text { Automatic Detection }\end{array}$} & \multicolumn{2}{|c|}{$\begin{array}{c}\text { SIT-TO-STAND } \\
\text { Automatic Detection }\end{array}$} \\
\hline & & not distressed & distressed & not distressed & distressed & not distressed & distressed \\
\hline \multirow{2}{*}{ 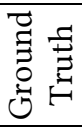 } & not distressed & $13(100 \%)$ & $0(0 \%)$ & $36(97.3 \%)$ & $1(2.7 \%)$ & $86(95.6 \%)$ & $4(4.4 \%)$ \\
\hline & distressed & $1(33.3 \%)$ & $2(66.7 \%)$ & $3(37.5 \%)$ & $5(62.5 \%)$ & $6(27.3 \%)$ & $16(72.7 \%)$ \\
\hline
\end{tabular}

For the Forward Trunk Flexion, unlike the other two activity types, all the features extracted were useful. Similar to the Full Trunk Flexion, the right lower leg energy feature was the most important feature; the leg speed feature almost matched this feature in importance. It is not clear why the leg speed was found to be important in this activity type but not in Full Trunk Flexion, which is a similar movement with respect to the legs.

For the Sit-to-Stand, the minimum hand to thigh distance was the most important feature for this activity type although, as can be seen in Fig. 4, about half of the not distressed instances had values similar to the values of the distressed instances. This is not surprising as in the Sit-to-Stand, there are reasons other than distress why the hands may be close to the thigh (e.g. natural resting of the hands on the thigh at the beginning of the activity).

6.2.2 Linear Mixed Model Analysis. Distressed participants were found to have significantly lower energy of the left and right shank in Full and Forward Trunk Flexion $(p<.05)$. These participants also had significantly lower minimum hand-to-thigh distance in Full Trunk Flexion $(p<.01)$ and Sit-to-Stand $(p<.005)$ suggesting that they used thigh self-adaptors in these movement types. Further, the distressed participants were found to be significantly slower $(p<.05)$ in Forward Trunk Flexion and Sit-to-Stand. An unexpected finding was that the distressed had significantly higher minimum head angle $(p<.05)$ in both Forward Trunk Flexion and Sit-to-Stand. It may be that pain related distress lends to rigidity in the movement of the head that masks head slump typical of distress (in non-pain contexts). 
Full Trunk Flexion - Not Distressed versus Distressed
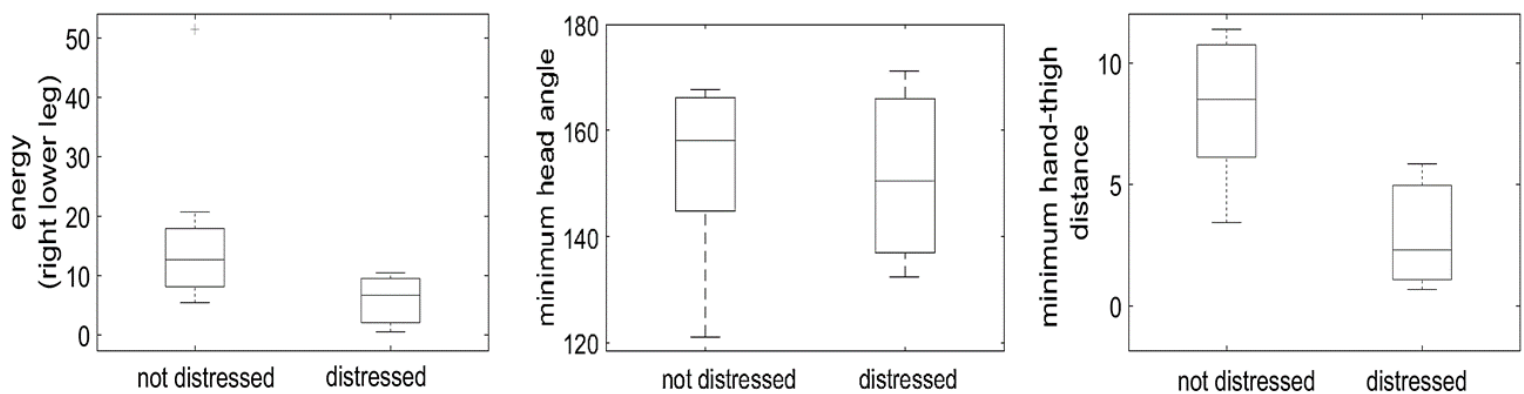

Fig. 3. Left - Right lower leg energies. Middle - Minimum head angles. Right - Minimum hand-thigh distances.

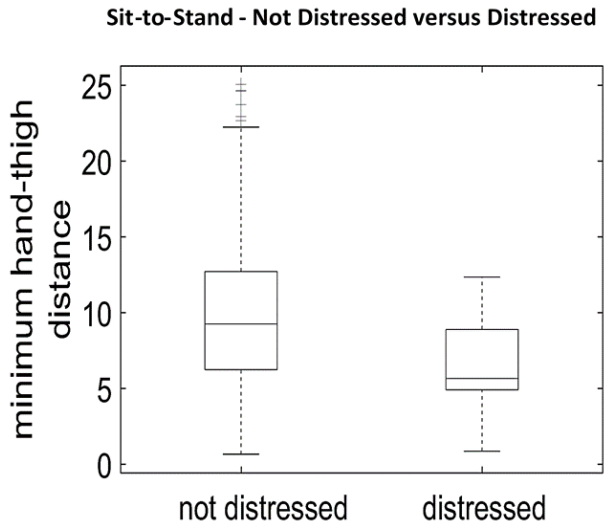

Fig. 4. Minimum hand-thigh distances.

\section{STUDY 3 - AUTOMATIC DETECTION OF PAIN LEVELS - RESULTS}

In this section, we first report the performance of the automatic detection of three pain groups (no CP, lower level pain, and higher level pain) based on the exercise movements of the EmoPain dataset and the relevance of the selected features (see Section 5.2) to this task. These have been previously reported in [16-17] but are described here for completeness including new results on the relevance of the features based on linear mixed model analysis. We then report completely new results of automatic detection of lower and higher level pain groups in both functional and exercise movements based on the Ubi-EmoPain dataset [63]. The latter is a critical step to understand the feasibility of moving into everyday functioning and how the informative qualities of movement transfer between exercise and functional movements.

\subsection{Results: Detection in Exercise Movements (based on Commercial Sensors)}

7.1.1 Classification Performance. As can been seen in Table 3, pain level detection using the optimised feature set was better than chance level classification for the three activity types. Detection in Full Trunk Flexion was perfect; this may be due to the relatively small size of the dataset and so lower random inter-subject variation in contrast to the other two exercises. The SVM performed at least as good as the RF for the three activity types. Table 4 shows the confusion matrices for classification for each activity type with this algorithm. 
Table 3. Pain Level Classification Performance with Exercise Movements captured using Commercial Sensors (with the Optimised Features based on Feature Subset Selection)

\begin{tabular}{ccccccc}
\hline & \multicolumn{2}{c}{ Full Trunk Flexion } & \multicolumn{2}{c}{ Forward Trunk Flexion } & \multicolumn{2}{c}{ Sit-to-Stand } \\
& SVM & RF & SVM & RF & SVM & RF \\
\hline F1 no CP & 1 & 1 & $\mathbf{0 . 9 5}$ & 0.77 & $\mathbf{0 . 8 5}$ & 0.73 \\
F1 lower level pain & 1 & 1 & $\mathbf{0 . 8 4}$ & 0.59 & $\mathbf{0 . 7 7}$ & 0.58 \\
F1 higher level pain & 1 & 1 & $\mathbf{0 . 7 6}$ & 0.56 & $\mathbf{0 . 9 0}$ & 0.74 \\
average F1 & 1 & 1 & $\mathbf{0 . 8 5}$ & 0.64 & $\mathbf{0 . 8 4}$ & 0.68 \\
accuracy & 1 & 1 & $\mathbf{0 . 8 6}$ & 0.65 & $\mathbf{0 . 8 5}$ & 0.68 \\
\hline
\end{tabular}

Table 4. Confusion Matrices for The Best Classifier in Table 3 (i.e. the SVM) for Each Activity Type

\begin{tabular}{|c|c|c|c|c|}
\hline \multicolumn{5}{|c|}{ FULL TRUNK FLEXION (SVM) } \\
\hline & & \multicolumn{3}{|c|}{ Automatic Detection } \\
\hline & & no $\mathrm{CP}$ & lower level pain & higher level pain \\
\hline \multirow{3}{*}{ 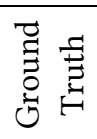 } & no $\mathrm{CP}$ & 6 & 0 & 0 \\
\hline & lower level pain & 0 & 7 & 0 \\
\hline & higher level pain & 0 & 0 & 5 \\
\hline \multicolumn{5}{|c|}{ FORWARD TRUNK FLEXION (SVM) } \\
\hline & & \multicolumn{3}{|c|}{ Automatic Detection } \\
\hline & & no $\mathrm{CP}$ & lower level pain & higher level pain \\
\hline \multirow{3}{*}{ 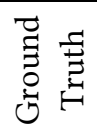 } & no $\mathrm{CP}$ & $17(89.4 \%)$ & $1(5.3 \%)$ & $1(5.3 \%)$ \\
\hline & lower level pain & $0(0 \%)$ & $13(86.7 \%)$ & $2(13.3 \%)$ \\
\hline & higher level pain & $0(0 \%)$ & $3(20 \%)$ & $12(80 \%)$ \\
\hline \multicolumn{5}{|c|}{ SIT-TO-STAND (SVM) } \\
\hline & & \multicolumn{3}{|c|}{ Automatic Detection } \\
\hline & & no $\mathrm{CP}$ & lower level pain & higher level pain \\
\hline \multirow{3}{*}{ 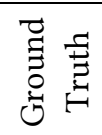 } & no $\mathrm{CP}$ & $34(87.2 \%)$ & $3(7.7 \%)$ & $2(5.1 \%)$ \\
\hline & lower level pain & $6(20 \%)$ & $22(73.3 \%)$ & $2(6.7 \%)$ \\
\hline & higher level pain & $1(2.9 \%)$ & $2(5.7 \%)$ & $32(91.4 \%)$ \\
\hline
\end{tabular}

7.1.2 Feature Subset Selection. Our analysis showed that the range of trunk movement was useful in differentiating between a person with $\mathrm{CP}$ and a healthy person during Full and Forward Trunk Flexion, although this feature was not useful in discriminating between lower and higher level pain within the CP group. Similarly, even though visual exploration had shown arm unsteadiness to be a possible cue of pain level within the $\mathrm{CP}$ group, it was only found to be useful in differentiating the healthy from the $\mathrm{CP}$ group in these two activity types. Amount of neck flexion was found to be useful in discriminating between lower and higher level pain in Forward Trunk Flexion but not for Full Trunk Flexion even though it was found useful in separating instances from healthy participants and instances from people with $\mathrm{CP}$ in the two activity types. Muscle relaxation pattern features were found to be important for differentiation of the three pain classes for the Full and Forward Trunk Flexion.

For Sit-to-Stand, the muscle relaxation pattern and the mean muscle activity features were of less importance than the kinematic features for discrimination between instances from healthy participants and 
those from participants with CP. However, for separating instances with lower level pain from those with higher level pain, these muscle activity features were important. We found that information about the relation between the seat height and the subject height was necessary for discriminating between the three pain levels, whereas the speed of lift was not at all useful.

7.1.3 Linear Mixed Model Analysis. $\quad$ For Full Trunk Flexion, significant difference $(p<.05)$ was only found for the activity change point of the right lumbar paraspinal and trapezius between participants with $\mathrm{CP}$ who reported higher level pain and healthy control participants. The result shows that the change point occurred significantly earlier in the lumbar paraspinal for the participants with higher level pain. However, the lower amount of relaxation in this muscle for the participants, although not significant, suggests that their premature relaxation may be due to either suboptimal flexion which enabled early extension and relaxation or suboptimal relaxation with complete relaxation delayed. The amount of relaxation was found to be significantly lower in the trapezius for the participants adding strength to this theory.

For Forward Trunk Flexion, difference was significant for the activity change point for both the left and right lumbar paraspinal and trapezius $(p<.05)$ in addition to the range of trunk and head motion $(p<.005)$. The result shows that the amount of trunk flexion and the displacement of the head was significantly lowest in participants with higher level pain and highest in healthy control participants. Like in Full Trunk Flexion, activity change point occurred in the right lumbar paraspinal significantly earlier in participants with higher level than in healthy control participants; in this case, the lower amount of change that occurred in participants with higher level pain was significant. These findings make a strong case for the earlier proposed theory although the significantly lower range of trunk flexion in this movement type specifically points to suboptimal flexion as the (perhaps unintentional) strategy that enabled early relaxation. There was also significantly lower relaxation of the left lumbar paraspinal and trapezius for the participants with higher level pain than for the healthy control participants.

The duration, speed of lift, range of trunk flexion, knee angles at lift, and activity features of the left lumbar paraspinal and trapezius were significantly different for the pain levels in Sit-to-Stand. Interestingly, participants with lower level pain had the lowest duration for the movement, lower than for healthy control participants despite healthy control participants significantly having the highest lift and extension speed $(p<.005)$. The additional finding of significantly higher amount of trunk flexion in the healthy control participants $(p<.005)$ suggests that although participants with lower level pain were slower in lift and extension, lower amount of trunk flexion led to lesser time to prepare for lift and possibly also lesser time to complete extension. Participants with higher level pain had significantly higher duration $(p<.05)$, lower speed $(p<.005)$, and lower range of trunk flexion $(p<.005)$ than either of the healthy control participants or the participants with lower level pain. It was also found that participants with lower level pain had significantly lower left and right knee angles $(p<.01$ and $p<.05$ respectively) just before lift than participants with higher level pain. This suggests that pain intensity may affect the positioning of the feet for standing up from seated position with those with higher level pain likely to keep their feet as far forward as possible. Another finding was that the activity change point of the left lumbar paraspinal occurred significantly earlier $(p<.005)$ in participants with higher level pain than in participants with lower level pain. As with Forward Trunk Flexion, this may be due to suboptimal flexion of the trunk and as expected, the range of activity of the muscle was also significantly lower $(p<.05)$ in participants with higher level pain than in participants with lower level pain.

\subsection{Results: Detection in Exercise and Functional Movements (based on Low-Cost Sensors)}

Here, we found that pain level detection was better with imputation and feature optimisation based on feature subset selection and was well above chance level classification (see Table 5). Detection for the Full and Forward Trunk Flexion without imputation was much lower than chance classification; this is likely due to the higher proportion of instances with missing values in these activity types: $67.4 \%$ and $53.3 \%$ respectively, compared to $52.9 \%$ for the Sit-to-Stand. This may also be a reason for the lower classification performance for the Full Trunk Flexion with imputation and optimisation compared with the other two activity types. The 
classification performance for the exercise and functional subsets were found to be comparable within each activity type with accuracies of 0.67 and 0.68 respectively for the Full Trunk Flexion and accuracies of 0.80 and 0.86 respectively for the Forward Trunk Flexion. The confusion matrices of classification based on the optimised feature sets are shown in Table 6.

Table 5. Pain Level Classification in Both Exercise and Functional Movements based on A Custom-Built Low-Cost Prototype

\begin{tabular}{|c|c|c|c|c|c|c|}
\hline \multicolumn{7}{|c|}{ UNOPTIMISED FEATURE SET } \\
\hline & \multicolumn{2}{|c|}{ Full Trunk Flexion } & \multicolumn{2}{|c|}{ Forward Trunk Flexion } & \multicolumn{2}{|c|}{ Sit-to-Stand } \\
\hline & Incomplete & Imputed & Incomplete & Imputed & Incomplete & Imputed \\
\hline & Dataset & Dataset & Dataset & Dataset & Dataset & Dataset \\
\hline F1 low level pain & 0.52 & 0.62 & 0.23 & 0.68 & 0.82 & 0.84 \\
\hline F1 high level pain & 0.32 & 0.35 & 0.34 & 0.60 & 0.67 & 0.72 \\
\hline average $\mathrm{F} 1$ & 0.42 & 0.49 & 0.29 & 0.64 & 0.75 & 0.78 \\
\hline accuracy & 0.43 & 0.52 & 0.31 & 0.64 & 0.76 & 0.79 \\
\hline \multicolumn{7}{|c|}{ OPTIMISED FEATURE SET (IMPUTED DATASET ONLY) } \\
\hline & \multicolumn{2}{|c|}{ Full Trunk Flexion } & \multicolumn{2}{|c|}{ Forward Trunk Flexion } & \multicolumn{2}{|c|}{ Sit-to-Stand } \\
\hline F1 low level pain & \multicolumn{2}{|c|}{0.71} & \multicolumn{2}{|c|}{0.86} & \multicolumn{2}{|c|}{0.86} \\
\hline F1 high level pain & \multicolumn{2}{|c|}{0.63} & \multicolumn{2}{|c|}{0.83} & \multicolumn{2}{|c|}{0.75} \\
\hline average F1 & \multicolumn{2}{|c|}{0.67} & \multicolumn{2}{|c|}{0.85} & \multicolumn{2}{|c|}{0.81} \\
\hline accuracy & \multicolumn{2}{|c|}{0.67} & \multicolumn{2}{|c|}{0.84} & \multicolumn{2}{|c|}{0.82} \\
\hline
\end{tabular}

Table 6. Confusion Matrices using Imputed Datasets with Optimised Feature Sets

\begin{tabular}{|c|c|c|c|}
\hline \multicolumn{4}{|c|}{ FULL TRUNK FLEXION } \\
\hline \multirow{4}{*}{ 忢 } & \multirow{4}{*}{$\begin{array}{l}\text { lower level pain } \\
\text { higher level pain }\end{array}$} & \multicolumn{2}{|c|}{ Automatic Detection } \\
\hline & & lower level pain & higher level pain \\
\hline & & $18(66.7 \%)$ & $9(33.3 \%)$ \\
\hline & & $6(31.6 \%)$ & $13(68.4 \%)$ \\
\hline \multicolumn{4}{|c|}{ FORWARD TRUNK FLEXION } \\
\hline \multirow{2}{*}{ 莺 } & lower level pain & $21(87.5 \%)$ & $3(12.5 \%)$ \\
\hline & higher level pain & $4(19.0 \%)$ & $17(\mathbf{8 1 . 0 \%})$ \\
\hline \multicolumn{4}{|c|}{ SIT-TO-STAND } \\
\hline \multirow{2}{*}{ 莺 } & lower level pain & $19(82.6 \%)$ & $4(17.4 \%)$ \\
\hline & higher level pain & $2(18.2 \%)$ & $9(\mathbf{8 1 . 8 \%})$ \\
\hline
\end{tabular}

\section{OVERALL DISCUSSION}

The investigations in this paper aimed to provide a deeper understanding of the opportunities of affectawareness functionality in healthcare technology, with a focus on CP physical rehabilitation. First, through qualitative analysis of existing interview data, we investigated how pain and related fear/anxiety and 
low/depressed mood interfere with engagement in physical activity of people with CP and how technological support may be personalised to address these affective barriers. Further, after making a case for movement behaviour as a critical window on these barriers, we explored automatic detection of emotional distress (combined fear/anxiety and low/depressed mood) and pain from movement behaviour and muscle activity during physical activity, as a primary step toward implementation of the proposed strategies. Our automatic recognition results demonstrated F1 score of 0.86 and 0.9 (average for three activity types) for two-level emotional distress classification and three-level pain classification respectively for exercise movements captured using full-body commercial sensors. We additionally provided preliminary evidence of F1 score of 0.78 (average for three activity types) for two-level pain classification for exercise and functional movements captured using a minimal set of low-cost sensors. In this section, we bring together the findings of the qualitative study and the results of the quantitative analysis in a discussion that aims to provide direction toward affect-based personalisation in $\mathrm{CP}$ physical rehabilitation technology.

\subsection{What Design Opportunities Does Affect Awareness Introduce?}

Our findings in Study 1 suggest two design opportunities that affect awareness can enable in technological support for physical rehabilitation:

8.1.1 Personalising Encouragement. Existing principles suggest elements of 'positive reinforcement' in design aimed at increasing engagement in physical activity [87]. Our findings in Study 1 further suggest two standards to follow in providing encouragement in the case of physical rehabilitation for long-term medical conditions. First, it is important that encouragement is timely. In the case of $\mathrm{CP}$ physical rehabilitation, encouragement is needed in periods of increased pain when the tendency is to avoid physical activities even when valued. Encouragement should additionally be provided when these periods are prolonged and there is the danger of psychological setbacks. Periods of low mood also call for encouragement.

Further, encouragement provided must be more than simply presenting recorded data (e.g. physical activity levels) to the user: it must be rewarding. For people with $\mathrm{CP}$ where gains in physical capability are slow and setbacks more likely, periods where encouragement may most be needed, data on current physical performance may not be encouraging. In this case, data from previous recoveries from setbacks and gains in psychological capability can provide encouragement [88]. It may be necessary to embed the data within a message of reassurance.

Awareness of levels of the relevant affect state based on automatic detection equip technology for such targeted support.

8.1.2 Regulating Awareness. Several physical activity intervention approaches, e.g. in [19][89-90], are built on enabling awareness. Further, exergame-based designs, shown to enable improvement in motor skills of people with CP [91] incorporate awareness [10]. What we propose is the regulation of such awareness to relevant affective experiences. For people with $\mathrm{CP}$, awareness needs to be provided (or its saliency increased) at appropriate times on low level pain days to prompt breaks; appropriate times may be determined by a person's average exertion level given that physiotherapists with expertise in CP suggest gradual exposure to physical activity [11]. Such regulation in time may instead be based on goals set by the person with $\mathrm{CP}$, to give him/her control [11][87]. A person with CP additionally needs to recognise instances when pain related fear/anxiety or low mood leads to maladaptive strategies or behaviours. Some of these instances will be best addressed in real time (especially for fear/anxiety) with more helpful strategies immediately suggested [92], e.g. deep breathing to reduce tension. Although people are taught some of these strategies during pain management programs, as found in our analysis, they also need (just-in-time) reminders.

Other instances may require awareness on reflection, for the purposes of altering pain beliefs and informing self-planned strategies for engaging in (challenging) physical activities. In fact, pain management programs aim to (re-)educate a person with CP and equip her/him with a toolbox of strategies that s/he can adapt to address barriers to engagement in physical activity [93]. Personal (affect as well as movement behaviour) data can inform reflective (re-)formulation [19]. However, it is again important that data is not simply presented to the user. While it is necessary to ensure transparency and data availability, some level of 
abstraction serves to protect the user from unhelpful reflection. For people with $\mathrm{CP}$, for example, care must be taken not to direct attention to pain levels, or (unconsciously) associate movement with pain. Yet, it is important that available data are as comprehensive as possible. For people with $\mathrm{CP}$, movement behaviour data needs to be provided with data on levels of fear/anxiety and mood to support useful reflection. Continuous tracking of affective states together with concomitant body movement enable technology to address this requirement. Automatic tracking can generally support the regulation of awareness [19][94].

\subsection{How Then Can Technology Assess Affective Barriers?}

We have set an example in this paper that the design of affect awareness in healthcare technology should be based on understanding of the affective needs of the population that it is designed for. The literature on pain behaviour points to movement behaviour as the most relevant modality for insight into pain and related states and our results in Studies 2 and 3 indicate that this modality should be strongly considered in developing affect-awareness capability for CP physical rehabilitation technology. Performance using this modality is comparable with state-of-the-art performance based on facial expressions (e.g. [5][7-8]), although fair comparison is difficult as most of these studies focus on the detection of observer-rated expressions of pain rather than self-report (the clinical standard for assessing pain intensity [71]). An additional merit of movement behaviour as a modality in this context is that existing physical rehabilitation technologies would typically be already endowed with body movement sensors making it possible for them to acquire affect tracking capabilities. While we focused on the use of wearable sensors in our investigation, our findings can easily transfer to other body movement sensor technologies used in $\mathrm{CP}$ physical rehabilitation, such as optical-based sensors [91]. A significant contribution of our findings is development of the understanding of movement behaviour cues, which can be used by developers of physical rehabilitation technology to equip it with affect recognition functionality toward implementation of the design principles discussed in the previous section. Clinicians similarly depend on behavioural cues to infer relevant mental states (e.g. pain-related selfefficacy [63]) and decide what support to provide to the specific individual during that specific period [11]. Further, these cues can be used by the technology as suggestions of what to look for to read the action tendency of or strategies used by a person experiencing pain or distress, e.g. notice of unnecessary muscle tension in a person with high levels of fear during movement execution. As highlighted in the previous section, such information can be used to suggest more helpful strategies and/or help the person understand, via reflection, the influence of fear/anxiety or low/depressed mood on movement.

Our analysis of movement behaviour and muscle activity features provides deeper understanding of known movement behaviour cues of emotional distress and pain. For example, one of our findings was that the tempo of movement execution enables recognition of pain-related emotional distress similar to its usefulness in nonpain contexts [76][78]. This cue was also shown to enable automatic classification of pain levels in people with $\mathrm{CP}$, in addition to its use in differentiation of people with $\mathrm{CP}$ from healthy subjects [79]. We found that for pain, the duration of movement execution and movement speed may provide different descriptions of movement tempo. We hypothesize that the participants with $\mathrm{CP}$ achieved shorter duration of sit-to-stand movement despite lower speed in the ascension phase due to reduced trunk flexion (for ascension initiation), which enables less preparation for ascension and less adjustment to complete the extension phase of the movement.

Another finding was the relevance of the range of movement of the affected anatomical segment to the classification of pain levels within people with $\mathrm{CP}$ without the need to capture intricate motion features using delicate capture methods as was done in [47]. We also showed that head and shoulder droop can be cues of emotional distress in physical activity settings although this depends on the movement type: certain movement types may mask it (or make it salient). We found that unlike other contexts [72-74], with pain, while distressed people may keep their heads at minimum inclination for longer periods than those not distressed, they tend to have lower inclination values.

In addition, we showed that body sway characterised by joint energy enables recognition of pain-related emotional distress. It is difficult to compare our findings with studies in non-pain contexts because of 
differences in emotional distress constructs (as well body sway computation). This cue was analysed in [76] for depressed mood alone and lateral body sway was found to increase with depressed mood. In [77], body sway was considered with respect to anxiety alone, and anterior-posterior body sway was found to increase with anxiety but only for low frequency sway, with decrease with anxiety for high frequency sway.

Further, we provide insight to cues not previously studied in the literature. For example, we showed that the range of head or neck movement enables discrimination between levels of pain. Although this cue may be related to guarding behaviour (i.e. stiffness in movement), it should be noted that the head and neck were not the main pain locations of the participants with CP (although some of the participants may have had pain in these segments in addition to the low back). Another cue discovered in our investigation was arm unsteadiness for automatically differentiating pain classes, although no statistical significance was found. These two findings suggest that, for $\mathrm{CP}$, stiffness in movement may generalise beyond the painful segment.

It was also shown that pain leads to atypical foot positioning to perform sit-to-stand movement. We found that people with higher level pain keep their feet as far forward as possible to initiate the movement and use upper limbs to lift the body in order to execute this biomechanically challenging strategy. Our data showed two ways in which the upper limbs were used: (1) both hands are used to push up from the bench, or (2) one side (usually the right side) of the body is raised before the other in ascension and the hand on the contralateral side is used to push up from the bench.

Further, we showed that back muscle relaxation on trunk re-extension can contribute to discrimination between levels of pain with pain leading to earlier relaxation. We believe that this is due to the reduced tension in flexion (in turn due to lower trunk flexion) that is more likely with (higher levels of) pain.

Finally, we showed that the use of thigh self-adaptors may be a cue of emotional distress in physical activity settings. This finding builds on the work of [73] on other types of self-adaptors in sedentary settings.

\subsection{Is Affect-Aware Personalisation for CP Physical Rehabilitation Feasible in Everyday Settings?}

The possibility of technology-based support for CP physical rehabilitation in everyday functioning has been explored in [19] and [94]. As discussed in the introduction, the shift in physical rehabilitation settings from constrained (exercise sessions) to day-to-day has been found to be necessary to enable the transfer of strategies and capabilities learnt in the former, and also to support the integration of exercise into everyday functioning to promote adherence to prescribed exercises [18-19]. Aligned with this shift, we have shown the possibility of tracking pain levels during both exercise and functional movements using a minimal network of low-cost body movement sensors. This is important groundwork towards the leveraging of affect-based personalisation during day-to-day activities.

Our findings call for more work led by both the HCI and Affective Computing communities. It is particularly necessary to further evaluate how technological intervention with the proposed design strategies integrated can help people with $\mathrm{CP}$ to better manage engagement in physical activities. For example, how does regulating awareness to fear/anxiety level (e.g. providing a reminder to a person with $\mathrm{CP}$ to breathe deeply while $\mathrm{s} / \mathrm{he}$ is completing a movement where $\mathrm{s} / \mathrm{he}$ is exhibiting fear responses) help a person with $\mathrm{CP}$ engage in the activities that they find challenging during everyday functioning? And what awareness designs are helpful? Although studies like [89] address the latter concern, few have considered functional activity settings for psychologically demanding medical conditions like CP [93]. The available literature suggests unobtrusiveness as a factor that will be critical to tackle in such designs [87][95]. Perhaps more interaction between the fitness and the healthcare technology industries can encourage designs that address both physical rehabilitation and fitness needs, and so normalise the use of physical rehabilitation technology, minimising the stigma associated with the use of healthcare aids in everyday life [96]. The prevalence of chronic illnesses needing long-term physical rehabilitation indeed makes this a practical consideration.

To fully explore these problems, further work needs to be done on continuous tracking of pain, fear/anxiety, and mood in unsegmented activity where the boundary between one movement and the next may be non-existent. For instance, a person may reach for a distant object (and so perform forward trunk flexion) at the same time as standing up from seated position (a sit-to-stand). One possible approach to 
addressing this challenge is to focus on components of movements (e.g. flexion of the trunk) independent of the type of movement(s) involved (e.g. full and forward trunk flexion or sit-to-stand). This approach additionally provides a convenient solution to the problem of understanding the action intention of the person, for the dual purpose of informing the strategy to be suggested towards easier completion of the movement (when that is appropriate) and as supplementary information for the affect detection module.

It is also important to understand how the users of the technology manage its imperfections, for example, false positives and negatives in automatic detection. How can these imperfections be made transparent to the user within its design? How do the users appropriate such information? The findings of Verame et al. [97] suggest that transparency can help the users understand how to incorporate feedback from an imperfect system; however, the constant burden of managing $\mathrm{CP}$ may present unexpected consideration for people with CP.

\section{CONCLUSIONS}

Our work addresses the need for affect-based personalisation in $\mathrm{CP}$ physical rehabilitation. First, based on the analysis of interview data that reflect perspectives of people with $\mathrm{CP}$ and pain-specialist physiotherapists, we provide deeper understanding of how pain and related fear/anxiety and low/depressed mood individually interfere with functioning. Further, based on the findings from this analysis, we propose two design strategies that address the barriers that these states pose. One of these design principles is the personalisation of encouragement so that it is provided just-in-time in the event of relevant affective states or experiences, and additionally based on rewarding information, which may be of affective experiences. The other principle is the regulation of awareness (e.g. of affective response) to the relevant affective experiences. Both strategies assume automatic affect detection capability. Indeed, we provide understanding of how movement behaviour and muscle activity features can be a window on pain and related emotional distress (i.e. combined fear/anxiety and low/depressed mood). We show that these features enable good levels of discrimination (average F1 score $=0.9$ and 0.86 for two and three levels of pain and emotional distress, respectively). We further provide evidence that pain levels can be automatically detected based on a minimal set of low-cost body movement sensors for ubiquitous monitoring ( 0.78 average F1 score for two levels). We highlight directions for future work in this area.

\section{ACKNOWLEDGMENTS}

This work was funded by the EPSRC grant Emotion \& Pain Project EP/H017178/1 and Olugbade was supported by the 2012 Nigerian Presidential Special Scholarship Scheme for Innovation and Development (PRESSID). 
A THE COMPUTATION OF EMOTIONAL DISTRESS FEATURES

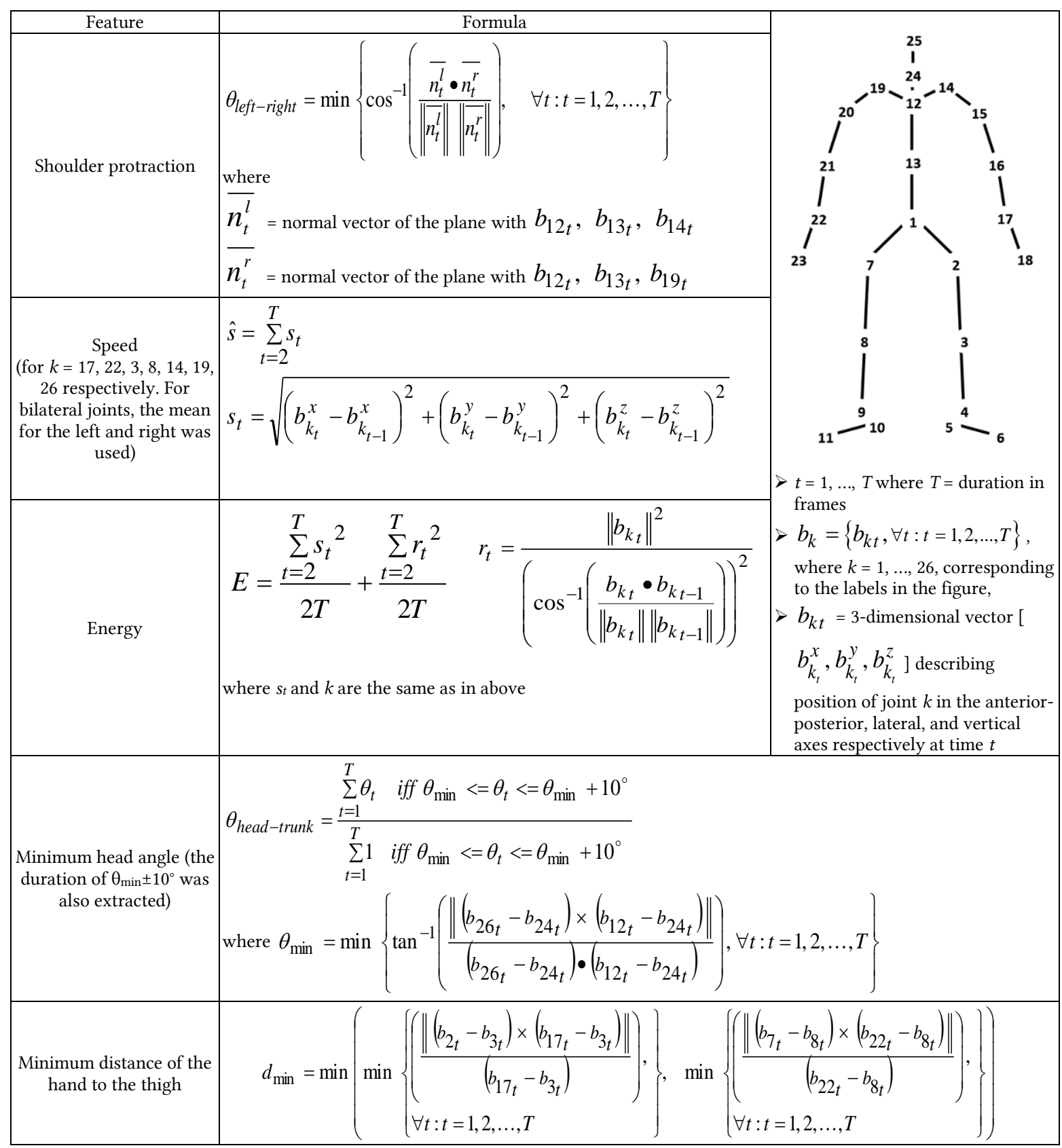


B THE COMPUTATION OF PAIN LEVEL FEATURES

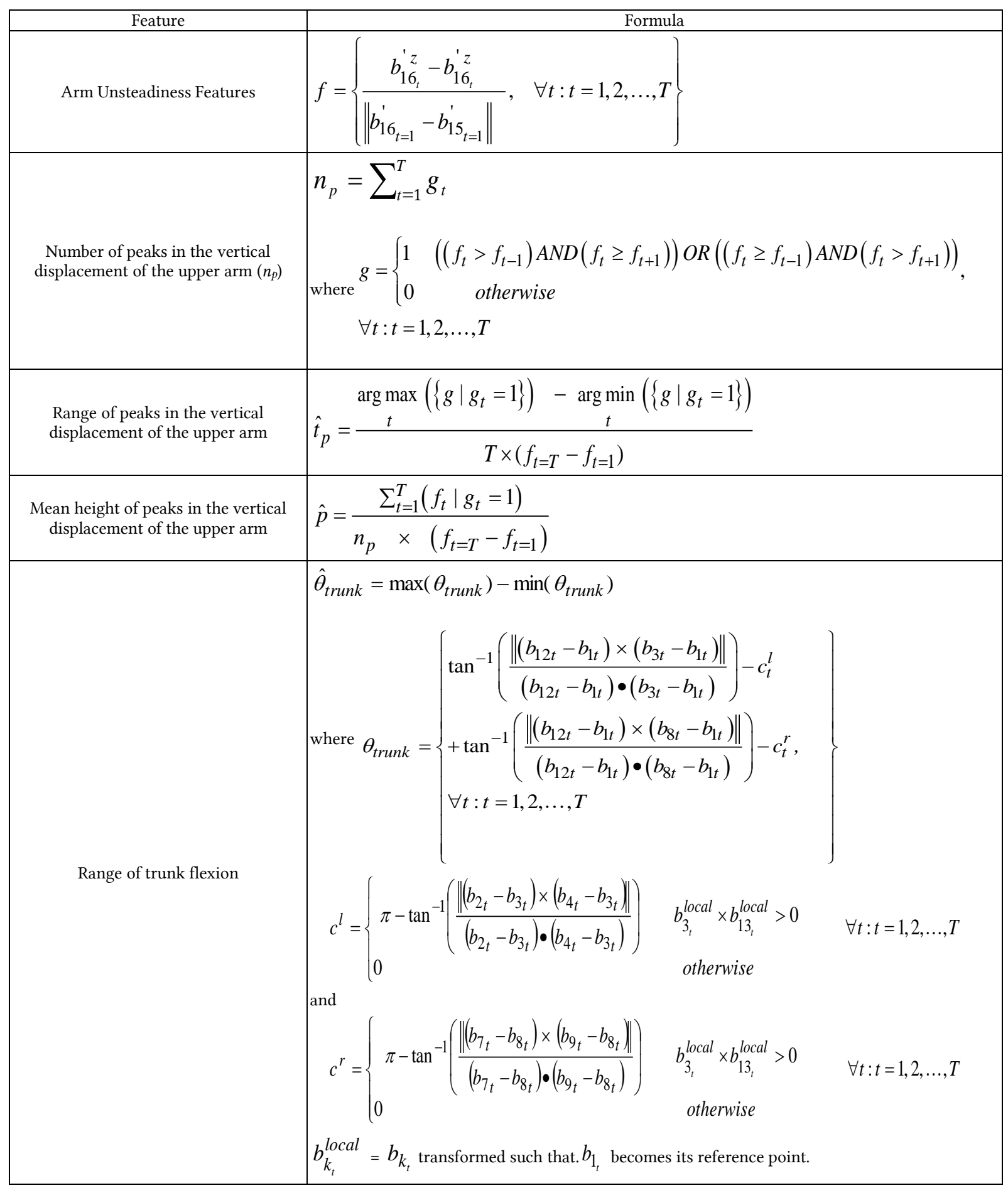




\begin{tabular}{|c|c|}
\hline Feature & Formula \\
\hline $\begin{array}{l}\text { Range of trunk flexion } \\
\text { (with respect to the ground) }\end{array}$ & 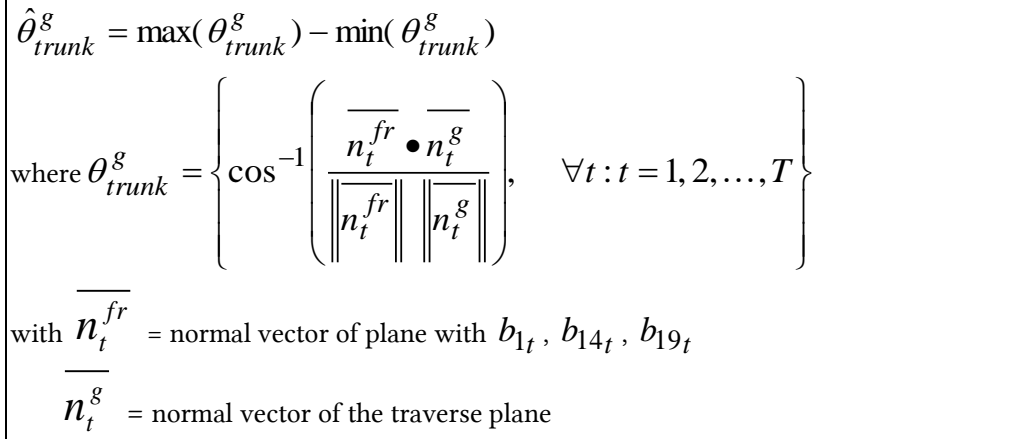 \\
\hline $\begin{array}{l}\text { Range of trunk flexion before lift } \\
\text { (for sit-to-stand) }\end{array}$ & $\begin{array}{l}\hat{\theta}_{\text {trunk }}^{\text {lift }}=\max \left(\theta_{\text {trunk }}^{\text {lift }}\right)-\min \left(\theta_{\text {trunk }}^{\text {lift }}\right) \\
\theta_{\text {trunk }}^{\text {lift }}=\left\{\begin{array}{l}\tan ^{-1}\left(\frac{\left\|\left(b_{12 t}-b_{1 t}\right) \times\left(b_{3 t}-b_{1 t}\right)\right\|}{\left(b_{12 t}-b_{1 t}\right) \bullet\left(b_{3 t}-b_{1 t}\right)}\right)+\tan ^{-1}\left(\frac{\left\|\left(b_{12 t}-b_{1 t}\right) \times\left(b_{8 t}-b_{1 t}\right)\right\|}{\left(b_{12 t}-b_{1 t}\right) \bullet\left(b_{8 t}-b_{1 t}\right)}\right) \\
\forall t: t=1,2, \ldots, t\end{array}\right) \\
\text { where } t^{\text {lift }} \text { is the time the pelvic joint begins ascension. }\end{array}$ \\
\hline Range of head flexion & $\begin{array}{l}\hat{z}=\max (z)-\min (z) \\
z=\left\{\frac{b_{24_{t}}^{z}-b_{13_{t}}^{z}}{\left\|b_{24_{t=1}}-b_{13_{t=1}}\right\|}, \quad \forall t: t=1,2, \ldots, T\right\}\end{array}$ \\
\hline $\begin{array}{l}\text { Muscle relaxation change point } \\
\quad(\text { for each of muscle } q)\end{array}$ & $\hat{t}_{e}=\frac{\underset{t}{\arg \max }\left(\left\{\frac{\sum_{i=t-(w+d / 2)}^{t-d / 2} m_{q_{i}}-\frac{\sum_{i=t+d / 2}^{t+(w+d / 2)}}{w} m_{q_{i}}}{w}, \quad \forall t: t=w+(d+1) / 2, \ldots, T-(w+(d+1) / 2)\right\}\right)}{T}$ \\
\hline $\begin{array}{l}\text { Muscle relaxation magnitude } \\
\quad \text { (for each of muscle } q \text { ) }\end{array}$ & $\left.\left.\hat{\operatorname{enax}}=\frac{\min \left(\left\{\frac{\sum_{i=t-(w+d / 2)}^{t-d / 2} m_{q_{i}}}{w}-\frac{\sum_{i=t+d / 2}^{t+(w+d / 2)} m_{q_{i}}}{w}, \quad \forall t: t=w+(d+1) / 2, \ldots, T-(w+(d+1) / 2)\right\}\right.}{\max \left(m_{q}\right)}\right)\right\}$ \\
\hline $\begin{array}{l}\text { Duration } \\
\text { (for sit-to-stand) }\end{array}$ & $\begin{array}{l}\Delta t=T^{*}-t_{1} \\
\text { where } T^{*}=\text { frame where pelvic joint reaches maximum vertical displacement }\end{array}$ \\
\hline $\begin{array}{l}\text { Average lift speed } \\
\text { (for sit-to-stand) }\end{array}$ & $\hat{s}=\frac{\sum_{t=t}^{T^{*}} \text { lift } s_{t}}{T} \quad$ where $s=\left\{b_{1_{t}}^{z}-b_{1_{t-1}}^{z}, \quad \forall t: t=2,3, \ldots, T\right\}$ \\
\hline
\end{tabular}




\begin{tabular}{|c|c|}
\hline Feature & Formula \\
\hline Angle at lift & 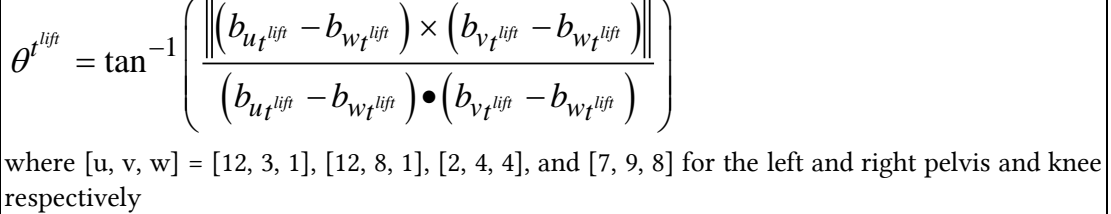 \\
\hline
\end{tabular}

\section{REFERENCES}

[1] Jyoti Joshi, Abhinav Dhall, Roland Goecke, and Jeffrey F. Cohn. 2013. Relative body parts movement for automatic depression analysis. In Proceedings of ACII, 492-497.

[2] Jyoti Joshi, Roland Göecke, Sharifa Alghowinem, Abhinav Dhall, Michael Wagner, Julien Epps, Gordon Parker, and Michael Breakspear. 2013. Multimodal Assistive Technologies for Depression Diagnosis and Monitoring. F Multimodal User Interfaces 7, 3 , $217-28$.

[3] Jyoti Joshi, Roland Göecke, Gordon Parker, and Michael Breakspear. 2013. Can Body Expressions Contribute to Automatic Depression Analysis? In Proceedings of FG, 1-7.

[4] David DeVault, Ron Artstein, Grace Benn, Teresa Dey, Ed Fast, Alesia Gainer, Kallirroi Georgila, Jon Gratch, Arno Hartholt, Margaux Lhommet, Gale Lucas, Stacy Marsella, Fabrizio Morbini, Angela Nazarian, Stefan Scherer, Giota Stratou, Apar Suri, David Traum, Rachel Wood, Yuyu Xu, Albert Rizzo, and Louis-Philippe Morency. 2014. SimSensei kiosk: a virtual human interviewer for healthcare decision support. In Proceedings of AAMAS, 1061-1068.

[5] Neeru Rathee and Dinesh Ganotra. 2016. Multiview Distance Metric Learning on facial feature descriptors for automatic pain intensity detection. Comput Vision Image Underst 147, 77-86.

[6] George Michael, Kyriakos Tsaparellas, Gabriel Panis, Christos P. Loizou, and Andreas Lanitis. 2016. Towards Non-invasive Patient Monitoring Through Iris Tracking and Pain Detection. In Proceedings of Mediterranean Conference on Medical and Biological Engineering and Computing 361-366.

[7] Philipp Werner, Ayoub Al-Hamadi, Kerstin Limbrecht-Ecklundt, Steffen Walter, Sascha Gruss, and Harald Traue. 2016. Automatic pain assessment with facial activity descriptors. IEEE T Affect Comput.

[8] Sourav Dey Roy, Mrinal Kanti Bhowmik, Priya Saha, and Anjan Kumar Ghosh. 2016. An Approach for Automatic Pain Detection through Facial Expression. Procedia Computer Science 84, 99-106.

[9] Jing Zhou, Xiaopeng Hong, Fei Su, and Guoying Zhao. 2016. Recurrent Convolutional Neural Network Regression for Continuous Pain Intensity Estimation in Video. In Proceedings of ICVR, 84-92.

[10] Christian Schönauer, T. Pintaric, H. Kaufmann, S. Jansen-Kosterink, and M. Vollenbroek-Hutten. 2011. Chronic pain rehabilitation with a serious game using multimodal input. In Proceedings of ICVR, 1-8.

[11 Aneesha Singh, Annina Klapper, Jinni Jia, Antonio Fidalgo, Ana Tajadura-Jiménez, Natalie Kanakam, Nadia Bianchi-Berthouze, and

] Amanda Williams. 2014. Motivating people with chronic pain to do physical activity: opportunities for technology design. In Proceedings of CHI, 2803-2812.

[12] Johan W. S. Vlaeyen, Stephen Morley, and Geert Crombez. 2016. The experimental analysis of the interruptive, interfering, and identity-distorting effects of chronic pain. Behav Res Ther 86, 23-34

[13 Ali Asghari and Michael K. Nicholas. 2001. Pain self-efficacy beliefs and pain behaviour. A prospective study. Pain 94, 1, 85-100.

]

[14 Dennis Turk. 2015. Generalization and Maintenance of Performance. Fordyce's Behavioural Methods for Chronic Pain and Illness. C. ] Main, F. Keefe, M. Jensen, J. Vlaeyan, K. Vowles, Eds. IASP Press Washington, D. C., 415-427.

[15] Michael J. L. Sullivan, Pascal Thibault, André Savard, Richard Catchlove, John Kozey, and William D. Stanish. 2006. The influence of communication goals and physical demands on different dimensions of pain behavior. Pain 125, 3, 270-277.

[16 Temitayo Olugbade, M. Aung, N. Bianchi-Berthouze, N. Marquardt, and A. Williams. 2014. Bi-modal detection of painful reaching for ] chronic pain rehabilitation systems. In Proceedings of ICMI, 455-458.

[17] Temitayo A. Olugbade, Nadia Bianchi-Berthouze, Nicolai Marquardt, and Amanda C. Williams. 2015. Pain level recognition using kinematics and muscle activity for physical rehabilitation in chronic pain. In Proceedings of ACII, 243-249.

[18] Naveen L. Bagalkot, Tomas Sokoler, and Suraj Baadkar. 2016. Reride: performing lower back rehabilitation while riding your motorbike in traffic. In Proceedings of PervasiveHealth, 77-80.

[19 Aneesha Singh, Nadia L. Berthouze-Bianchi, and A. Williams. 2017. Supporting Everyday Function in Chronic Pain Using a Wearable ] Device. In Proceedings of $\mathrm{CHI}$.

[20] Harald Breivik, Beverly Collett, Vittorio Ventafridda, Rob Cohen, and Derek Gallacher. 2006. Survey of chronic pain in Europe: Prevalence, impact on daily life, and treatment. Eur f Pain 10, 4, 287-333.

[21] Catherine Johannes, T. Kim Le, X. Zhou, J. Johnston, and R. Dworkin. 2010. The prevalence of chronic pain in United States adults: results of an Internet-based survey. J Pain 11, 11, 1230-1239.

[22] Irene Tracey and M. Catherine Bushnell. 2009. How neuroimaging studies have challenged us to rethink: is chronic pain a disease? $\mathcal{F}$ Pain 10, 11, 1113-1120.

[23 The Pain Consortium. 2016. UK Pain Messages. Pain News.

]

ACM Trans. Comput.-Hum. Interact. xx, xx, Article xx (Month xxx) 
[24 Eva Denison, P. Åsenlöf, and P. Lindberg. 2004. Self-efficacy, fear avoidance, and pain intensity as predictors of disability in subacute ] and chronic musculoskeletal pain patients in primary health care. Pain 111, 3, 245-252.

[25] Johan W. S. Vlaeyen and Steven J. Linton. 2000. Fear-avoidance and its consequences in chronic musculoskeletal pain: a state of the art. Pain 85, 3, 317-332.

[26 Sara M. Banks and Robert D. Kerns. 1996. Explaining High Rates of Depression in Chronic Pain: A Diathesis-Stress Framework. ] Psychological Bulletin 119, 1, 95-110.

[27] Adina Rusu, Tamar Pincus, and Stephen Morley. 2012. Depressed pain patients differ from other depressed groups: examination of cognitive content in a sentence completion task. Pain 153, 9, 1898-1904.

[28] Virginia Braun and V. Clarke. 2006. Using thematic analysis in psychology. Qual Res Psychol 3, 2, 77-101.

[29 Vicki Harding and Paul J. Watson. 2000. Increasing activity and improving function in chronic pain management. Physiotherapy 86, ] 12, 619-630.

[30 Chris Eccleston. 2001. Role of psychology in pain management. Br f Anaesth 87, 1, 144-152.

]

[31 Zina Trost, Karoline Vangronsveld, Steven J. Linton, Phillip J. Quartana, and Michael J. L. Sullivan. 2012. Cognitive dimensions of ] anger in chronic pain. Pain 153, 3, 515-517.

[32 Tamar Pincus, Rob Smeets, Maureen Simmonds, and Michael Sullivan. 2010. The fear avoidance model disentangled: improving the ] clinical utility of the fear avoidance model. Clin f Pain 26, 9, 739-746.

[33 Angela S. Lee, Jacek Cholewicki, N. Peter Reeves, Bohdanna T. Zazulak, and Lawrence W. Mysliwiec. 2010. Comparison of trunk ] proprioception between patients with low back pain and healthy controls. Arch Phys Med Rehabil 91, 9, 1327-1331.

[34 Andrea Kleinsmith and Nadia Bianchi-Berthouze. 2013. Affective body expression perception and recognition: A survey. IEEE T ] Affect Comput 4, 1, 15-33.

[35 Hatice Gunes, Caifeng Shan, Shizhi Chen, and YingLi Tian. 2015. Bodily expression for automatic affect recognition. Emotion ] Recognition: A Pattern Analysis Approach (1st ed.) Amit Konar and Aruna Chakraborty, Eds. Wiley and Sons, 343-377.

[36 Min S. H. Aung, Sebastian Kaltwang, Bernardino Romera-Paredes, Brais Martinez, Aneesha Singh, Matteo Cella, Michel Valstar ] Hongying Meng, Andrew Kemp, Moshen Shafizadeh, Aaron C. Elkins, Natalie Kanakam, Amschel de Rothschild, Nick Tyler, Paul J. Watson, Amanda C. de C. Williams, Maja Pantic, and Nadia Bianchi-Berthouze. 2016. The automatic detection of chronic painrelated expression: requirements, challenges and the multimodal EmoPain dataset. IEEE T Affect Comput 7, 4, 435-451.

[37 Rafael A. Calvo and Sidney D'Mello. 2010. Affect detection: An interdisciplinary review of models, methods, and their applications. ] IEEE T Affect Comput 1, 1, 18-37.

[38 Paul J. Watson, C. Kerry Booker, and Chris J. Main. 1997. Evidence for the role of psychological factors in abnormal paraspinal ] activity in patients with chronic low back pain. 7 Musculoskelet Pain 5, 4, 41-56.

[39 Paul J. Watson, C. K. Booker, Chris J. Main, and A. C. N. Chen. 1997. Surface electromyography in the identification of chronic low ] back pain patients: the development of the flexion relaxation ratio. Clin Biomech 12, 3, 165-171.

[40 Steffen Walter, Sascha Gruss, Kerstin Limbrecht-Ecklundt, Harald C. Traue, Philipp Werner, Ayoub Al-Hamadi, Nicolai Diniz,

] Gustavo Moreira da Silva, and Adriano O. Andrade. 2014. Automatic pain quantification using autonomic parameters. Psychol Neurosci 7, 3, 363.

[41 Markus Kächele, Mohammadreza Amirian, Patrick Thiam, Philipp Werner, Steffen Walter, Günther Palm, and Friedhelm Schwenker.

] 2016. Adaptive confidence learning for the personalization of pain intensity estimation systems. Evolving Systems, 1-13.

[42 Valéry Legrain, Stefaan Van Damme, Christopher Eccleston, Karen D. Davis, David A. Seminowicz, and Geert Crombez. 2009. A ] neurocognitive model of attention to pain: behavioral and neuroimaging evidence. Pain 144, 3, 230-232.

[43 S. Pearce, S. Isherwood, D. Hrouda, P. Richardson, A. Erskine, and J. Skinner. 1990. Memory and pain: tests of mood congruity and ] state dependent learning in experimentally induced and clinical pain. Pain 43, 2, 187-193.

[44 David K. Ahern, Michael J. Follick, James R. Council, Nancy Laser-Wolston, and Henry Litchman. 1988. Comparison of lumbar ] paravertebral EMG patterns in chronic low back pain patients and non-patient controls. Pain 34, 2, 153-160.

[45 Helena Grip, Fredrik Ohberg, Urban Wiklund, Ylva Sterner, J. Stefan Karlsson, and Björn Gerdle. 2003. Classification of neck ] movement patterns related to whiplash-associated disorders using neural networks. IEEE Trans Inf Technol Biomed 7, 4, 412-418.

[46 Daniel T. H. Lai, Pazit Levinger, Rezaul K. Begg, Wendy Lynne Gilleard, and Marimuthu Palaniswami. 2009. Automatic recognition ] of gait patterns exhibiting patellofemoral pain syndrome using a support vector machine approach. IEEE Trans Inf Technol Biomed $13,5,810-817$.

[47 James P. Dickey, Michael R. Pierrynowski, Drew A. Bednar, and Simon X. Yang. 2002. Relationship between pain and vertebral ] motion in chronic low-back pain subjects. Clin Biomech 17, 5, 345-352.

[48 Jeffrey F. Cohn, Tomas Simon Kruez, Iain Matthews, Ying Yang, Minh Hoai Nguyen, Margara Tejera Padilla, Feng Zhou, and ] Fernando De la Torre. 2009. Detecting depression from facial actions and vocal prosody. In Proceedings of ACII, 1-7.

[49 Sharifa Alghowinem, Roland Goecke, Michael Wagner, Gordon Parkerx, and Michael Breakspear. 2013. Head pose and movement ] analysis as an indicator of depression. In Proceedings of ACII, 283-288.

[50] Sharifa Alghowinem, Roland Goecke, Julien Epps, Michael Wagner, and Jeffrey Cohn. 2016. Cross-Cultural Depression Recognition from Vocal Biomarkers. Interspeech, 1943-1947.

[51] Anastasia Pampouchidou, Anastasia Pampouchidou, Olympia Simantiraki, Amir Fazlollahi, Matthew Pediaditis, Dimitrios Manousos, Alexandros Roniotis, Georgios Giannakakis, Fabrice Meriaudeau, Panagiotis Simos, Kostas Marias, Fan Yang, and Manolis Tsiknakis. 2016. Depression Assessment by Fusing High and Low Level Features from Audio, Video, and Text. AVEC, 27-34.

[52] Michel Valstar, Björn Schuller, Kirsty Smith, Florian Eyben, Bihan Jiang, Sanjay Bilakhia, Sebastian Schnieder, Roddy Cowie, and Maja Pantic. 2013. AVEC 2013: the continuous audio/visual emotion and depression recognition challenge. In Proceedings of AVEC, 310.

ACM Trans. Comput.-Hum. Interact. xx, xx, Article xx (Month xxx). 
[53 Md Nasir, Arindam Jati, Prashanth Gurunath Shivakumar, Sandeep Nallan Chakravarthula, and Panayiotis Georgiou. 2016. ] Multimodal and Multiresolution Depression Detection from Speech and Facial Landmark Features. In Proceedings of AVEC, 43-50.

[54 Le Yang, Dongmei Jiang, Lang He, Ercheng Pei, Meshia Cedric Oveneke, and Hichem Sahli. 2016. Decision Tree Based Depression ] Classification from Audio Video and Language Information. In Proceedings of AVEC, 89-96.

[55] Tamar Pincus and Stephen Morley. 2001. Cognitive-processing bias in chronic pain: a review and integration. Psych Bull $127,5,599$.

[56] Hongying Meng, Andrea Kleinsmith, and Nadia Bianchi-Berthouze. 2011. Multi-score learning for affect recognition: the case of body postures. In Proceedings of ACII, 225-234.

[57] Shizhi Chen, Y. Tian, Q. Liu, and D. Metaxas. 2013. Recognizing expressions from face and body gesture by temporal normalized motion and appearance features. Image Vis Comput 31, 2, 175-185.

[58] Stefano Piana, Alessandra Stagliano, Francesca Odone, Alessandro Verri, and Antonio Camurri. 2014. Real-time automatic emotion recognition from body gestures. arXiv.

[59] Ali-Akbar Samadani, Ali Ghodsi, and Dana Kulić. 2013. Discriminative functional analysis of human movements. Pattern Recognit Lett 34, 15, 1829-1839.

[60] Pramila Rani, Nilanjan Sarkar, and Changchun Liu. 2005. Maintaining Optimal Challenge in Computer Games through Real-Time Physiological Feedback. In Proceedings of CHI.

[61 Francis J. Keefe and Andrew R. Block. 1982. Development of an observation method for assessing pain behavior in chronic low back ] pain patients. Behav Ther 13, 4, 363-375.

[62 Aneesha, Singh, Stefano Piana, Davide Pollarolo, Gualtiero Volpe, Giovanna Varni, Ana Tajadura-Jiménez, Amanda C. de C.

] Williams, Antonio Camurri, and Nadia Bianchi-Berthouze. 2016. Go-with-the-flow: tracking, analysis and sonification of movement and breathing to build confidence in activity despite chronic pain. HCI 31, 3-4, 335-383.

[63 Temitayo A. Olugbade, Nadia Bianchi-Berthouze, Nicolai Marquardt, and Amanda C. Williams. 2018. Human Observer and

] Automatic Assessment of Movement Related Self-Efficacy in Chronic Pain: From Exercise to Functional Activity. IEEE Trans Affect Comput.

[64 Marc Thioux, Valeria Gazzola, and Christian Keysers. 2008. Action Understanding: How, What and Why. Current Biology 18, 10, ] R431-34.

[65] Wim G. M. Janssen, Hans BJ Bussmann, and Henk J. Stam. 2002. Determinants of the sit-to-stand movement: a review. Phys Ther 82, 9,866 .

[66 Anthony S. Zigmond and R. Philip Snaith. 1983. The hospital anxiety and depression scale. Acta Psychiatr Scand 67, 6, 361-370.

]

[67] Theodore D. Cosco, Frank Doyle, Mark Ward, and Hannah McGee. 2012. Latent structure of the Hospital Anxiety and Depression Scale: a 10-year systematic review. F Psychosom Res 72, 3, 180-184.

[68] Adina C. Rusu, Rita Santos, and Tamar Pincus. 2016. Pain-related distress and clinical depression in chronic pain: A comparison between two measures. Scand I Pain 12, 62-67.

[69 Tamar Pincus, Amanda C. de C Williams, Steven Vogel, and Andy Field. 2004. The development and testing of the depression, ] anxiety, and positive outlook scale (DAPOS). Pain 109, 1, 181-188.

[70] Julie F. Pallant and Catherine M. Bailey. 2005. Assessment of the structure of the Hospital Anxiety and Depression Scale in musculoskeletal patients. Health Quality Life Outcomes 3, 1, 82

[71] Mark Jensen and P. Karoly. 1992. Self-report scales and procedures for assessing pain in adults. Handbook of Pain Assessment (2nd ed.). D. Turk and R. Melzack, Eds. Guilford Press, New York, 135-151.

[72] Peter Waxer. 1974. Nonverbal cues for depression. F Abnorm Psychol 83, 3, 319-322.

[73 Stefan Scherer, Giota Stratou, Marwa Mahmoud, Jill Boberg, Jonathan Gratch, Albert Rizzo, and Louis-Philippe Morency. 2013.

] Automatic behavior descriptors for psychological disorder analysis. FG, 1-8.

[74 Nesrine Fourati and Catherine Pelachaud. 2015. Relevant body cues for the classification of emotional body expression in daily ] actions. In Proceedings of ACII, 267-273.

[75] José Luís Pimentel do Rosário, Maria Suely Bezerra Diógenes, Rita Mattei, and José Roberto Leite. 2013. Can sadness alter posture? J Bodyw Mov Ther 17, 3, 328-331.

[76] Johannes Michalak, Nikolaus F. Troje, Julia Fischer, Patrick Vollmar, Thomas Heidenreich, and Dietmar Schulte. 2009. Embodiment of sadness and depression-gait patterns associated with dysphoric mood. Psychosom Med 71, 5, 580-587.

[77] M. Wada, N. Sunaga, and M. Nagai. 2001. Anxiety affects the postural sway of the antero-posterior axis in college students. Neurosci Lett 302, 2, 157-159.

[78] Matthias R. Lemke, Thomas Wendorff, Brigitt Mieth, Katharina Buhl, and Martin Linnemann. 2000. Spatiotemporal gait patterns during over ground locomotion in major depression compared with healthy controls. $\mathcal{F}$ Psychiatr Res 34, 4, 277-283.

[79] Gary Shum, Jack Crosbie, and Raymond Lee. 2005. Effect of low back pain on the kinematics and joint coordination of the lumbar spine and hip during sit-to-stand and stand-to-sit. Spine 30, 17, 1998-2004.

[80] G. Gioftsos and D. W. Grieve. 1996. The use of artificial neural networks to identify patients with chronic low-back pain conditions from patterns of sit-to-stand manoeuvres. Clin Biomech 11, 5, 275-280.

[81] D. E. Marple-Horvat and S. L. Gilbey. 1992. A method for automatic identification of periods of muscular activity from EMG recordings. 7 Neurosci Methods 42, 3, 163-167.

[82] Corinna Cortes and Vladimir Vapnik. 1995. Support-vector networks. Machine Learning 20, 3, 273-297.

[83 Leo Breiman. 2001. Random forests. Machine Learning 45, 1, 5-32.

]

[84 Leo Breiman, Jerome H. Friedman, Richard A. Olshen, and Charles J. Stone. 1984. Classification and regression trees. Wadsworth \& 
] Brooks. Monterey.

[85] Andy Field. 2013. Discovering Statistics Using IBM SPSS Statistics. 4th ed. SAGE Publications: London.

[86] Patrenahalli M. Narendra and Keinosuke Fukunaga. 1977. A branch and bound algorithm for feature subset selection. IEEE Trans Comput 26, 9, 917-922.

[87] Sunny Consolvo, David W. McDonald, and James A. Landay. 2009. Theory-driven design strategies for technologies that support behavior change in everyday life. In Proceedings of CHI, 405-414.

[88] Glenn Affleck, Howard Tennen, Alex Zautra, Susan Urrows, Micha Abeles, and Paul Karoly. 2001. Women's pursuit of personal goals in daily life with fibromyalgia: A value-expectancy analysis. 7 Consult Clin Psychol 69, 4, 587.

[89] Kristina Höök, Martin Jonsson, Anna Ståhl, and Johanna Mercurio. 2016. Somaesthetic Appreciation Design. In Proceedings of CHI, 3131-3142.

[90] Filippo Casamassima, Alberto Ferrari, Bojan Milosevic, Pieter Ginis, Elisabetta Farella, and Laura Rocchi. 2014. A wearable system for gait training in subjects with Parkinson's disease. Sensors 14, 4, 6229-6246.

[91 Stephanie M. Jansen-Kosterink, Rianne M. H. A. Huis in’t Veld, Christian Schönauer, Hannes Kaufmann, Hermie J. Hermens, and ] Miriam MR Vollenbroek-Hutten. 2013. A serious exergame for patients suffering from chronic musculoskeletal back and neck pain: a pilot study. Games Health f 2, 5, 299-307.

[92 Sergio Felipe, Aneesha Singh, Caroline Bradley, Amanda C. de C. Williams, and Nadia Bianchi-Berthouze. 2015. Roles for personal ] informatics in chronic pain. In Proceedings of PervasiveHealth, 161-168.

[93 Dennis C. Turk and Akiko Okifuji. 2002. "Psychological Factors in Chronic Pain: Evolution and Revolution." f Consul Clin Psychol 70, ] 3: $678-90$

[94 Geoffrey B. Duggan, Edmund Keogh, Gail A. Mountain, Paul McCullagh, Jason Leake, and Christopher Eccleston. 2015. Qualitative ] evaluation of the SMART2 self-management system for people in chronic pain. Disabil Rehabil Assist Technol 10, 1, 53-60.

[95] Enrica Papi, Athina Belsi, and Alison H. McGregor. 2015. A knee monitoring device and the preferences of patients living with osteoarthritis: a qualitative study. BM7 Open 5, 9, e007980.

[96 Aisling Ann O'Kane, Yvonne Rogers, and Ann E. Blandford. 2015. Concealing or revealing Mobile medical devices?: designing for ] onstage and offstage presentation. In Proceedings of CHI, 1689-1698.

[97] Jhim Kiel M. Verame, Enrico Costanza and Sarvapali D. Ramchurn. 2016. The Effect of Displaying System Confidence Information on the Usage of Autonomous Systems for Non-specialist Applications: A Lab Study. In Proceedings of CHI, 4908-4920.

ACM Trans. Comput.-Hum. Interact. xx, xx, Article xx (Month xxx). 